\title{
THE MUTUAL INDUCTANCE OF TWO CIRCULAR COAXIAL COILS OF RECTANGULAR SECTION.
}

\author{
By Edward B. Rosa and Louis Cohen.
}

Various formulæ have been proposed from time to time for calculating the mutual inductance of coaxial coils. All of them are approximate formulæ and in most cases the approximation is closer if the coils are not near each other. The degree of approximation is not, however, shown by the formulæ themselves, and it is therefore highly important to critically examine and compare all the formulæ available, and to ascertain which are most accurate and what the magnitude of the residual error is likely to be in any given case. A practical question, for example, is to determine what limitations as to size of section, radius, and distance apart must be placed on two coils in order that their mutual inductances may be computed to one part in 50,000. If such coils are to be used in the absolute measurement of resistance, the formulæ employed for computing the mutual inductance must be justified beyond question. We propose in this paper to examine these formulæ and to compare them by numerous numerical calculations. We shall show which are the more accurate formulæ, point out where some of them fail, and shall derive some new expressions more convenient to use than some of those which have heretofore been employed. We shall also give a number of examples to illustrate and test the formulæ, and curves to show the relative accuracy of various formulæ for particular coils at varying distances. 


\section{MUTUAL INDUCTANCE OF TWO COAXIAL CIRCLES.}

MAXWELL'S FORMULF IN ELLIPTIC INTEGRALS.

Some of the formulæ available give the mutual inductance of two coaxial coils directly in terms of the dimensions of the coils, while others derive it from the mutual inductance of two coaxial circles, either by giving the correction to be applied to the latter, or by employing in the formula for the latter a modified radius, or by combining several values for circles in such a way as to give (approximately) the value for coils of appreciable cross section. It is therefore desirable to consider first the formulæ for the calculation of the mutual inductance of two coaxial circles. The first and most important is the formula in elliptic integrals given by Maxwell: ${ }^{1}$

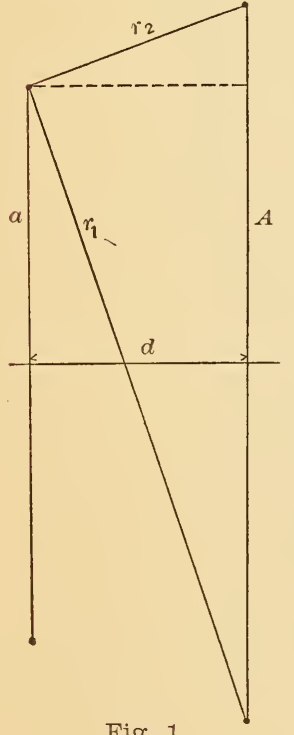

Fig. 1

$$
M=4 \pi \sqrt{A a}\left\{\left(\frac{2}{k}-k\right) F-\frac{2}{k} E\right\}
$$

in which $A$ and $a$ are the radii of the two circles, $d$ is the distance between their centers, and

$$
k=\frac{2 \sqrt{A a}}{\sqrt{ }(A+a)^{2}+d^{2}}=\sin \gamma
$$

$F$ and $E$ are the complete elliptic integrals of the first and second kind, respectively, to modulus $k$. Their values may be obtained from the tables of Legendre, or the values of $M \div 4 \pi \sqrt{A a}$ may be obtained from the table in Appendix I at the end of Chapter XIV (Vol. II) of Maxwell, the values of $\gamma$ being the argument.

The notation of Maxwell is slightly altered in the above expressions in order to bring it into conformity with the formulæ to follow.

Formula (I) is an absolute one, giving the mutual inductance of two coaxial circles of any size at any distance apart. If the two circles have equal or nearly equal radii, and are very near each other, the quantity $k$ will be very nearly equal to unity and $\gamma$ will be near 
to $90^{\circ}$. Under these circumstances it may be difficult to obtain a sufficiently exact value of $F$ and $E$ from the tables, as the quantities are varying rapidly and it is necessary to employ an interpolation formula to get values between those given in the tables. Under such circumstances the following formula, also given by Maxwell (derived by means of Landen's transformation), is more suitable:

$$
M=8 \pi \frac{\sqrt{A \alpha}}{\sqrt{k_{1}}}\left\{F_{1}-E_{1}\right\}
$$

in which $F_{1}$ and $E_{1}$ are complete elliptic integrals to modulus $k_{1}$, and

$$
k_{1}=\frac{r_{1}-r_{2}}{r_{1}+r_{2}}=\sin \gamma_{1}
$$

$r_{1}$ and $r_{2}$ are the greatest and least distances of one circle from the other (Fig. I); that is,

$$
\begin{aligned}
& r_{1}=\sqrt{(A+a)^{2}+d^{2}} \\
& r_{2}=\sqrt{(A-a)^{2}+d^{2}}
\end{aligned}
$$

The new modulus $k_{1}$ differs from unity more than $k$, hence $\gamma_{1}$ is not so near to $90^{\circ}$ as $\gamma$ and the values of the elliptic integrals can be taken more easily from the tables than when using formula (I) and the modulus $k$.

Another way of avoiding the difficulty when $k$ is nearly unity is to calculate the integrals $F$ and $E$ directly, and thus not use the tables of elliptic integrals, expanding $F$ and $E$ in terms of the complementary modulus $k^{\prime}$, where $k^{\prime}=\sqrt{I-k^{2}}$. The expressions for $F$ and $E$ are very convergent when $k^{\prime}$ is small. For convenience of reference they are here given. An example will be given later to illustrate the use of these formulæ.

$$
\begin{aligned}
& F=\log \frac{4}{k^{\prime}}+\frac{I^{2}}{2^{2}} k^{\prime 2}\left(\log \frac{4}{k^{\prime}}-\frac{2}{\mathrm{I} \cdot 2}\right) \\
&+\frac{I^{2}}{2^{2}} \frac{3^{2}}{4^{2}} k^{\prime 4}\left(\log \frac{4}{k^{\prime}}-\frac{2}{\mathrm{I} \cdot 2}-\frac{2}{3 \cdot 4}\right) \\
&+\frac{\mathrm{I}^{2}}{2^{2}} \frac{3^{2}}{4^{2}} \frac{5^{2}}{6^{2}} k^{\prime 6}\left(\log \frac{4}{k^{\prime}}-\frac{2}{\mathrm{I} \cdot 2}-\frac{2}{3 \cdot 4}-\frac{2}{5 \cdot 6}\right) \\
&+\frac{\mathrm{I}^{2}}{2^{2}} \frac{3^{2}}{4^{2 \prime}} \frac{5^{2}}{6^{2}} \frac{7^{2}}{8^{2}} k^{\prime 8}\left(\log \frac{4}{k^{\prime}}-\frac{2}{\mathrm{I} \cdot 2}-\frac{2}{3 \cdot 4}-\frac{2}{5 \cdot 6}-\frac{2}{7 \cdot 8}\right) \\
&+. . \\
&
\end{aligned}
$$




$$
\begin{aligned}
E=\mathrm{I} & +\frac{\mathrm{I}}{2} k^{\prime 2}\left(\log \frac{4}{k^{\prime}}-\frac{\mathrm{I}}{\mathrm{I} \cdot 2}\right) \\
& +\frac{\mathrm{I}^{2}}{2^{2}} \frac{3}{4} k^{\prime 4}\left(\log \frac{4}{k^{\prime}}-\frac{2}{\mathrm{I} \cdot 2}-\frac{\mathrm{I}}{3 \cdot 4}\right) \\
& +\frac{\mathrm{I}^{2}}{2^{2}} \frac{3^{2}}{4^{2}} \frac{5}{6} k^{\prime 6}\left(\log \frac{4}{k^{\prime}}-\frac{2}{\mathrm{I} \cdot 2}-\frac{2}{3 \cdot 4}-\frac{\mathrm{I}}{5 \cdot 6}\right) \\
& +\frac{\mathrm{I}^{2}}{2^{2}} \frac{3^{2}}{4^{2}} \frac{5^{2}}{6^{2}} \frac{7}{8} k^{\prime 8}\left(\log \frac{4}{k^{\prime}}-\frac{2}{\mathrm{I} \cdot 2}-\frac{2}{3 \cdot 4}-\frac{2}{5 \cdot 6}-\frac{\mathrm{I}}{7 \cdot 8}\right) \\
& +. \cdot . \cdot . \cdot .
\end{aligned}
$$

\section{WEINSTEIN'S FORMULA.}

Weinstein $^{2}$ gives an expression for the mutual inductance of two coaxial circles, in terms of the complementary modulus $k^{\prime}$ used in the preceding series (3) and (4). That is, substituting the values of $F$ and $E$ given above in equation (I) we have Weinstein's equation, which is as follows:

$$
\begin{aligned}
M=4 \pi \sqrt{A \alpha}\left\{\left(\mathrm{I}+\frac{3}{4} k^{\prime 2}+\frac{33}{64} k^{\prime 4}+\frac{\mathrm{IO} 7}{256} k^{\prime 6}+\frac{59 \mathrm{I} 3}{\mathrm{I} 6384} k^{\prime 8}+.\right)\left(\log \frac{4}{k^{\prime}}-\mathrm{I}\right)\right. \\
\left.-\left(\mathrm{I}+\frac{\mathrm{I} 5}{\mathrm{I} 28} k^{\prime 4}+\frac{\mathrm{I} 85}{\mathrm{I} 536} k^{\prime 6}+\frac{7465}{65536} k^{\prime 8}+\ldots\right)\right\}
\end{aligned}
$$

Evidently this expression is rapidly convergent when $k^{\prime}$ is small, and hence will give an accurate value of $M$ when the circles are near each other. Otherwise formula (I) may be more accurate. An example will be given later to test the correctness of this formula.

\section{NAGAOKA'S FORMULE.}

Nagaoka ${ }^{3}$ has given formulæ for the calculation of the mutual inductance of coaxial circles, without the use of tables of elliptic integrals. These formulæ make use of Jacobi's $q$-series, which is very rapidly convergent. The first is to be used when the circles are not near each other, the second when they are near each other. Either may be employed for a considerable range of distances between the extremes, although the first is more convenient. The first formula is as follows: 


$$
\begin{aligned}
M & =\mathrm{I} 6 \pi^{2} \sqrt{A a} \cdot q^{\frac{3}{2}}(\mathrm{I}+\epsilon) \\
& =4 \pi \sqrt{A a}\left\{4 \pi q^{\frac{3}{2}}(\mathrm{I}+\epsilon)\right\}
\end{aligned}
$$

where $A$ and $a$ are the radii of the two circles. $\epsilon$ is a correction term which can be neglected when the circles are quite far apart.

$$
\begin{aligned}
& \epsilon=3 q^{4}-4 q^{6}+9 q^{8}-\mathrm{I} 2 q^{10}+\ldots . \\
& q=\frac{l}{2}+\left(\frac{l}{2}\right)^{5}+\mathrm{I} 5\left(\frac{l}{2}\right)^{9}+\ldots . \\
& l=\frac{\mathrm{I}-\sqrt{k^{\prime}}}{\mathrm{I}+\sqrt{k^{\prime}}} \quad k^{\prime}=\frac{r_{2}}{r_{1}}=\frac{\sqrt{(A-a)^{2}+d^{2}}}{\sqrt{(A+a)^{2}+d^{2}}}
\end{aligned}
$$

$d$ being the distance between the centers of the circles, and $k^{\prime}$ the complementary modulus occurring in equations (3), (4), and (5).

Nagaoka's second formula is as follows:

$$
\begin{aligned}
& M=4 \pi \sqrt{A \alpha} \cdot \frac{\mathrm{I}}{2\left(\mathrm{I}-2 q_{1}\right)^{2}}\left\{\log _{\frac{\mathrm{I}}{q_{1}}}\left[\mathrm{I}+8 q_{1}\left(\mathrm{I}-q_{1}+4 q_{1}^{2}\right)\right]-4\right\} \\
& q_{1}=\frac{l_{1}}{2}+2\left(\frac{l_{1}}{2}\right)^{5}+\mathrm{I} 5\left(\frac{l_{1}}{2}\right)^{9}+\ldots . \\
& l_{1}=\frac{\mathrm{I}-\sqrt{k}}{\mathrm{I}+\sqrt{k}} \quad k=\frac{2 \sqrt{A a}}{\sqrt{(A+a)^{2}+d^{2}}}
\end{aligned}
$$

$k$ is the modulus of equation (I), but is employed here to obtain the value of the $q$-series instead of the values of the elliptic integrals employed in (I). This formula is ordinarily simpler in use than it appears, because some of the terms in the expressions above are usually negligibly small.

Examples will be given later illustrating the use of these formulæ.

\section{MAXWELL'S SERIES FORMULA.}

Maxwel1 ${ }^{4}$ obtained an expression for the mutual inductance between two coaxial circles in the form of a converging series which is often more convenient to use than the elliptical integral formula, and when the circles are nearly of the same radii and relatively near each other the value given is generally sufficiently exact. In the following formula $a$ is the smaller of the two radii, $c$ is their 
difference, $A-\alpha, d$ is the distance apart of the circles as before, and $r=\sqrt{c^{2}+d^{2}}$. The mutual inductance is then

$$
\begin{aligned}
M= & 4 \pi a\left\{\log \frac{8 a}{r}\left(\mathrm{I}+\frac{c}{2 a}+\frac{c^{2}+3 d^{2}}{\mathrm{I} 6 a^{2}}-\frac{c^{3}+3 c d^{2}}{32 a^{3}}+\ldots\right)\right. \\
& \left.-\left(2+\frac{c}{2 a}-\frac{3 c^{2}-d^{2}}{\mathrm{I} 6 a^{2}}+\frac{c^{3}-6 c d^{2}}{48 a^{3}}-. .\right)\right\}
\end{aligned}
$$

When the two radii are equal, as is often the case in practice, the equation is considerably simplified, as follows:

$$
M=4 \pi a\left\{\log \frac{8 a}{d}\left(\mathrm{I}+\frac{3 d^{2}}{\mathrm{I} 6 a^{2}}\right)-\left(2+\frac{d^{2}}{\mathrm{I} 6 a^{2}}\right)\right\}
$$

The above formulæ (8) and (9) are sufficiently exact for very many cases, the terms omitted in the series being unimportant when $\frac{c}{a}$ and $\frac{d}{d}$ are small. For example, if $\frac{d}{a}$ is o.I, the largest term neglected in (9) is less than two parts in a million. If, however $d=a$, this term will be more than one per cent, and the formula will be quite inexact.

Coffin $n^{5}$ has extended Maxwell's formula (9) for two equal circles by computing three additional terms for each part of the expression. This enables the mutual inductance to be computed with considerable exactness up to $d=\alpha$. Formula (I) is exact, as stated abore, for all distances, and either it or (6) should be used in preference to (Io) when $d$ is large. Coffin's formula is as follows:

$$
\begin{aligned}
M=4 \pi a\left\{\log \frac{8 a}{d}\right. & \left(\mathrm{I}+\frac{3 d^{2}}{\mathrm{I} 6 a^{2}}-\frac{\mathrm{I} 5 d^{4}}{8 . \mathrm{I} 28 a^{4}}+\frac{35 d^{6}}{\mathrm{I} 28^{2} a^{6}}-\frac{\mathrm{I} 575 d^{8}}{2 . \mathrm{I} 28^{3} a^{8}}+\ldots\right) \\
& \left.-\left(2+\frac{d^{2}}{\mathrm{I} 6 a^{2}}-\frac{3 \mathrm{I} d^{4}}{\mathrm{I} 6 . \mathrm{I} 28 a^{4}}+\frac{247 d^{6}}{6.128^{2} a^{6}}-\frac{7795 d^{8}}{8 . \mathrm{I} 28^{3} d^{8}}+\ldots\right)\right\}(\text { (IO) }
\end{aligned}
$$

We have extended Maxwell's formula (8) for unequal circles by expanding the terms of formula(I) in series and substituting these series in (I). The series (3) and (4) involve $k^{\prime}$, where $k^{\prime 2}=\mathrm{I}-k^{2}$. Hence $\mathrm{I}+k^{2}=2-k^{2}$.

Also

$$
\begin{aligned}
& r=\sqrt{x^{2}+y^{2}} \\
& k^{2}=\frac{4 A a}{(A+a)^{2}+x^{2}}
\end{aligned}
$$

${ }^{5}$ Bulletin of Bureau of Standards, 2, p. II3; 1906. 


$$
\begin{aligned}
& k^{\prime 2}=\frac{x^{2}+y^{2}}{(2 a+y)^{2}+x^{2}} \\
& \frac{\sqrt{A \alpha}}{k}={ }_{2}^{\mathrm{I}} \sqrt{(2 a+y)^{2}+x^{2}}=a \sqrt{\mathrm{I}+\frac{y}{a}+\frac{x^{2}+y^{2}}{4 a^{2}}}
\end{aligned}
$$

Equation (I) is therefore equal to

$$
M=4 \pi a \sqrt{\mathrm{I}+\frac{y}{a}+\frac{x^{2}+y^{2}}{4 a^{2}}}\left\{\left(\mathrm{I}+k^{\prime 2}\right) F-2 E\right\}
$$

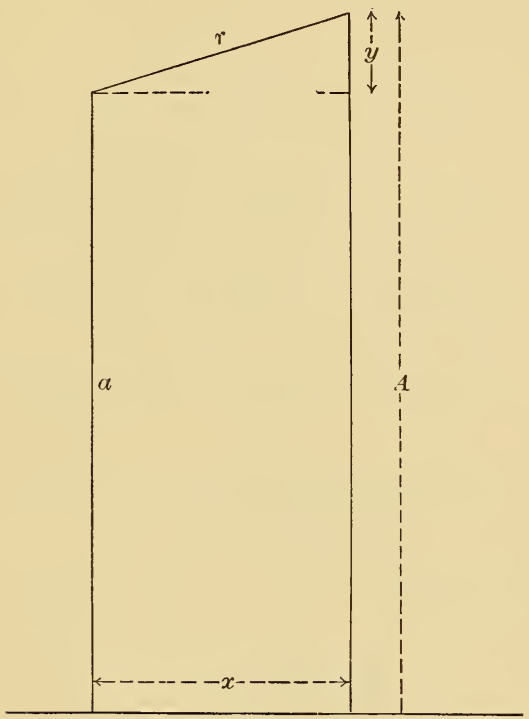

Fig. 2

Substituting the values of $F$ and $E$ from (3) and (4) we get

$$
\begin{aligned}
&\left\{\left(\mathrm{I}+k^{\prime 2}\right) F-2 E\right\}=\log \frac{4}{k^{\prime}}\left\{\mathrm{I}+\frac{k^{\prime 2}}{4}+\frac{k^{\prime 4}}{64}+\cdots\right\}-2+\frac{k^{\prime 2}}{4}-\frac{k^{\prime 4}}{\mathrm{I} 28}+\ldots \\
&=C \log \frac{4}{k^{\prime}}+D, \text { say } \\
& \log _{\frac{k^{\prime}}{4}}=\log \left(\frac{8 a}{r} \sqrt{\left(\mathrm{I}+\frac{y}{2 a}\right)^{2}+\frac{x^{2}}{4 a^{2}}}=\log \frac{8 a}{r}+\log \sqrt{\mathrm{I}+\frac{y}{2 a}+\frac{x^{2}+y^{2}}{4 a^{2}}}\right. \\
&=\log \frac{8 a}{r}+\frac{\mathrm{I}}{2}\left(\frac{y}{a}+\frac{x^{2}+y^{2}}{4 a^{2}}\right)-\frac{\mathrm{I}}{4}\left(\frac{y}{a}+\frac{x^{2}+y^{2}}{4 a^{2}}\right)^{2} \\
&+\frac{\mathrm{I}}{6}\left(\frac{y}{a}+\frac{x^{2}+y^{2}}{4 a^{2}}\right)^{3}-\ldots
\end{aligned}
$$

$16360-07-4$ 


$$
\text { Or, } \begin{aligned}
\log \frac{4}{k^{\prime}} & =\log \frac{8 a}{r}+\frac{y}{2 a}+\frac{x^{2}-y^{2}}{8 a^{2}}-\frac{6 x^{2} y-2 y^{3}}{48 a^{3}}-\frac{x^{4}-6 x^{2} y^{2}+y^{4}}{64 a^{4}} \\
& \quad+\frac{5 x^{4} y-10 x^{2} y^{3}+y^{5}}{\mathrm{I} 60 a^{5}} \\
& =\log \frac{8 a}{r}+B, \text { say } \\
k^{\prime 2} & =\frac{x^{2}+y^{2}}{4 a^{2}}\left(\mathrm{I}+\frac{y}{a}+\frac{x^{2}+y^{2}}{4 a^{2}}\right)^{-1} \\
& =\frac{x^{2}+y^{2}}{4 a^{2}}-\frac{x^{2} y+y^{3}}{4 a^{3}}-\frac{x^{4}-2 x^{2} y^{2}-3 y^{4}}{\mathrm{I} 6 a^{4}}+\frac{x^{4} y-y^{5}}{8 a^{5}} \ldots
\end{aligned}
$$

Substituting this value of $k^{\prime 2}$ above we have

$$
\begin{gathered}
C=\mathrm{I}+\frac{x^{2}+y^{2}}{\mathrm{I} 6 a^{2}}-\frac{x^{2} y+y^{3}}{\mathrm{I} 6 a^{3}}-\frac{\mathrm{I} 5 x^{4}-34 x^{2} y^{2}-49 y^{4}}{\mathrm{IO} 24 a^{4}} \\
+\frac{\mathrm{I} 5 x^{4} y-2 x^{2} y^{3}-\mathrm{I} 7 y^{5}}{5 \mathrm{I} 2 a^{5}}-\ldots \\
D=-2+\frac{x^{2}+y^{2}}{\mathrm{I} 6 a^{2}}-\frac{x^{2} y+y^{3}}{\mathrm{I} 6 a^{3}}-\frac{33^{4}-62 x^{2} y^{2}-95 y^{4}}{2048 a^{4}} \\
\quad+\frac{33 x^{4} y+2 x^{2} y^{3}-3 \mathrm{I} y^{5}}{\mathrm{IO} 24 a^{5}} \ldots \\
\left(\mathrm{I}+\frac{y}{a}+\frac{x^{2}+y^{2}}{4 a^{2}}\right)^{1 / 2}=\mathrm{I}+\frac{y}{2 a}+\frac{x^{2}}{8 a^{2}}-\frac{x^{2} y}{\mathrm{I} 6 a^{3}}+\frac{4 x^{2} y^{2}-x^{4}}{\mathrm{I} 28 a^{4}} \\
\quad+\frac{6 x^{4} y-8 x^{2} y^{3}}{5 \mathrm{I} 2 a^{5}}-\ldots=A, \text { say }
\end{gathered}
$$

Equation (a) is now

$$
M=4 \pi a \cdot A\left[C \log \frac{8 a}{r}+B C+D\right]
$$

in which $A, B, C$, and $D$ are the series $(g, c, e, f)$ given above. Substituting in $(h)$ and omitting terms of higher than the fifth degree we get

$$
\begin{aligned}
M & =4 \pi a\left\{\operatorname { l o g } \frac { 8 a } { r } \cdot \left(\mathrm{I}+\frac{y}{2 a}+\frac{3 x^{2}+y^{2}}{\mathrm{I} 6 a^{2}}-\frac{3 x^{2} y+y^{3}}{32 a^{3}}-\frac{\mathrm{I} 5 x^{4}-42 x^{2} y^{2}-\mathrm{I} 7 y^{4}}{\mathrm{IO} 24 a^{4}}\right.\right. \\
& \left.+\frac{45 x^{4} y-30 x^{2} y^{3}-\mathrm{I} 9 y^{5}}{2048 a^{5}}+\ldots\right)-\left(2+\frac{y}{2 a}+\frac{x^{2}-3 y^{2}}{\mathrm{I} 6 a^{2}}-\frac{6 x^{2} y-y^{3}}{48 a^{3}}\right. \\
& \left.\left.-\frac{93 x^{4}-534 x^{2} y^{2}-\mathrm{I} 9 y^{4}}{6 \mathrm{I} 44 a^{4}}+\frac{\mathrm{I} 845 x^{4} y-3030 x^{2} y^{3}-379 y^{5}}{6 \mathrm{I} 440 a^{5}}+\ldots\right)\right\}(\mathrm{I}
\end{aligned}
$$


When $y=0$, this gives the first part of series (Io). When $x=0$, the case of two circles in the same plane, with radii $a$ and $a+y$, we have

$$
\begin{array}{r}
M=4 \pi a\left\{\log \frac{8 a}{y}\left(\mathrm{I}+\frac{y^{\prime}}{2 a}+\frac{y^{2}}{\mathrm{I} 6 a^{2}}-\frac{y^{3}}{32 a^{3}}+\frac{\mathrm{I} 7 y^{4}}{\mathrm{IO} 24 a^{4}}-\frac{\mathrm{I} 9 y^{5}}{2048 a^{5}}-\ldots\right)\right. \\
\left.-\left(2+\frac{y}{2 a}-\frac{3 y^{2}}{\mathrm{I} 6 a^{2}}+\frac{y^{3}}{48 a^{3}}+\frac{\mathrm{I} 9 y^{4}}{6 \mathrm{I} 44 a^{4}}-\frac{379 y^{5}}{6 \mathrm{I} 440 a^{5}}-\ldots\right)\right\}
\end{array}
$$

In the above formulæ $x$ and $y$ are interchanged from Maxwell's notation and correspond to $d$ and $c$ of (8). That is, $x$ is the distance between the centers of the circles, and $y$ is the excess of one radius over $a$, the radius of the other; $y$ may be + or - .

These formulæ give the mutual inductance with great precision when the coils are not too far apart. The degree of convergence, of course, indicates in any case about what the limit of accuracy is.

We have derived equation (i I) also by the method given by Maxwell, ${ }^{6}$ to check the coefficients.

\section{MUTUAL INDUCTANCE OF TWO COAXIAL COILS.}

\section{ROWLAND'S FORMULA.}

Let there be two coaxial coils of mean radii $A$ and $a$, axial breadth of coils $b_{1}$ and $b_{2}$, radial depth $c_{1}$ and $c_{2}$, and distance apart of their mean planes $d$. Suppose them uniformly wound with $n_{1}$ and $n_{2}$ turns of wire. The mutual inductance $M_{0}$ of the two central turns, $\mathrm{O}_{1}$ and $\mathrm{O}_{2}$ (Fig. 3), will be given by formula (I) or (5), and the mutual inductance $M$ of the two coils of $n_{1}$ and $n_{2}$ turns will then be, to a first approximation,

$$
M=n_{1} n_{2} M_{0}
$$

A second approximation was obtained by Rowland by means of Taylor's theorem, following Maxwell, $\S 700$. The mutual inductance of the two central turns $\mathrm{O}_{1}$ and $\mathrm{O}_{2}$ being $M_{0}$, the mutual inductance of $\mathrm{O}_{1}$ on any turn at $\mathrm{P}$ of coordinates $x, y$ in coil $\mathrm{B}$ is given by 'Taylor's theorem, as follows: 


$$
M=M_{0}+x \frac{d M_{0}}{d x}+y \frac{d M_{0}}{d y}+\frac{x^{2} d^{2} M_{0}}{2}+\frac{y^{2}}{2} \frac{d^{2} M_{0}}{d y^{2}}+x y \frac{d^{2} M_{0}}{d x d y}+\ldots
$$

If we integrate this expression over the area of the coil $\mathrm{B}$ to find the equivalent value $M^{\prime}$ for the whole area, where

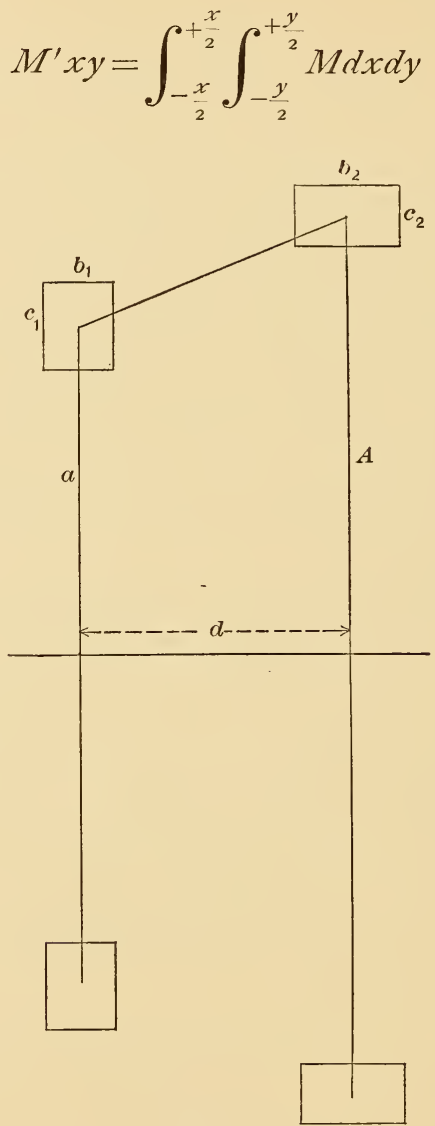

Fig. 3

we have (since the second and third terms become zero)

$$
M^{\prime}=M_{0}+\frac{x^{2}}{24} \frac{d^{2} M_{0}}{d x^{2}}+\frac{y^{2}}{24} \frac{d^{2} M_{0}}{d y^{2}}
$$

neglecting terms in $\frac{d^{4} M_{0}}{d x^{4}}, \frac{d^{4} M_{0}}{d x^{2} d y^{2}}$ and $\frac{d^{4} M_{0}}{d y^{4}}$ and higher orders. 
Substituting $b_{2}$ and $c_{2}$ for $x$ and $y$, and $d a$ for $d y$, we have

$$
M^{\prime}=M_{0}+\frac{b_{2}^{2}}{24} \frac{d^{2} M_{0}}{d x^{2}}+\frac{c_{2}^{2}}{24} \frac{d^{2} M_{0}}{d a^{2}}
$$

$n_{2} M^{\prime}$ is the mutual inductance of a single turn at $\mathrm{O}_{1}$ and the coil B. Repeating the process, integrating over $\mathrm{A}$, we get $M, n_{1} n_{2} M$ being the mutual inductance of one coil on the other. Thus,

$$
M=M_{0}+\frac{\mathrm{I}}{24}\left\{\left(b_{1}{ }^{2}+b_{2}{ }^{2}\right) \frac{d^{2} M_{0}}{d x^{2}}+c_{1}{ }^{2} \frac{d^{2} M_{0}}{d \alpha^{2}}+c_{2}{ }^{2} \frac{d^{2} M_{0}}{d A^{2}}\right\}
$$

If the two coils are of equal radii but unequal section,

$$
M=M_{0}+\frac{I}{24}\left\{\left(b_{1}^{2}+b_{2}^{2}\right) \frac{d^{2} M_{0}}{d x^{2}}+\left(c_{1}^{2}+c_{2}^{2}\right) \frac{d^{2} M_{0}}{d a^{2}}\right\}
$$

If the two coils are of equal radii and equal section, this becomes

$$
M=M_{0}+\frac{\mathrm{I}}{\mathrm{I} 2}\left\{b^{2} \frac{d^{2} M}{d x^{2}}+c^{2} \frac{d^{2} M}{d a^{2}}\right\}
$$

The value of $M_{0}$ should be calculated by formula (I). The correction terms will be calculated by means of the following:

$$
\begin{aligned}
& \frac{d^{2} M_{0}}{d a^{2}}=\pi \frac{k}{a}\left\{\left(2-k^{2}\right) F-\left(2-k^{2} \frac{\mathrm{I}-2 k^{2}}{\mathrm{I}-k^{2}}\right) E\right\} \\
& \frac{d^{2} M_{0}}{d x^{2}}=\pi \frac{k^{3}}{a}\left\{F-\frac{\mathrm{I}-2 k^{2}}{\mathrm{I}-k^{2}} E\right\}
\end{aligned}
$$

The equation (I4) is equivalent to Rowland's equation, where $2 \xi$ and $2 \eta$ are the breadth and depth of the section of the coil, instead of $b$ and $c$, except that there is an error in the formula as printed in Rowland's ${ }^{7}$ paper, $\xi$ and $\eta$ being interchanged. The equations (I 5) are equivalent to those given by Rowland, being somewhat simpler. ${ }^{8}$

Formula (I2) gives a very exact value for the mutual inductance of two coils, provided the cross sections are relatively small and the distance apart $d$ is not too small. But when $b$ or $c$ is large or $d$ is small the fourth differential coefficients which have been neglected become appreciable and the expression may not be sufficiently exact.

\footnotetext{
${ }^{7}$ Collected Papers, p. 162.

${ }^{8}$ Gray, Absolute Measurements, Vol. II, Part II, p. 322.
} 


\section{RAYLEIGH'S FORMULA.}

Maxwel1 ${ }^{9}$ gives a formkla, suggested by Rayleigh, for the mutual inductance of two coils, which has a very different form from Rowland's, but is nearly equivalent to it when the coils are not near each other. It has been 11sed by Glazebrook and Rayleigh, and may also be employed in calculating the attraction between two coils. $^{10}$ It is sometimes called the formula of quadratures. It is derived as follows:

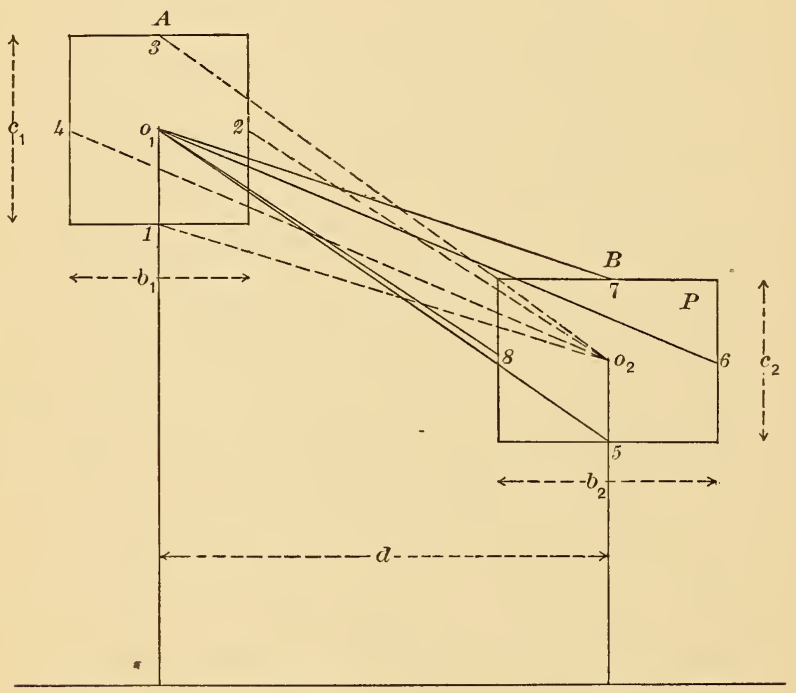

Fig. 4

Referring to Fig. 4, let $M_{1}, M_{3}$ be the mutual inductances of the central wire $\mathrm{O}_{2}$ and the wires at points I and 3 , respectively, of coil A. For these points $x=o$, and $y$ is $-\frac{c_{1}}{2}$ and $+\frac{c_{1}}{2}$, respectively. Substituting these values of $x$ and $y$ in equation (2) we have,

$$
\begin{aligned}
& M_{1}=M_{0}-\frac{c_{1}}{2} \frac{d M_{0}}{d a}+\frac{c_{1}{ }^{2}}{8} \frac{d^{2} M_{0}}{d a^{2}}-\frac{c_{1}{ }^{3}}{48} \frac{d^{3} M_{0}}{d a^{3}}+\frac{c_{1}{ }^{4}}{384} \frac{d^{4} M_{0}}{d a^{4}}-\ldots \\
& M_{3}=M_{0}+\frac{c_{1}}{2} \frac{d M_{0}}{d a}+\frac{c_{1}{ }^{2}}{8} \frac{d^{2} M_{0}}{d a^{2}}+\frac{c_{1}{ }^{3}}{48} \frac{d^{3} M_{0}}{d a^{3}}+\frac{c_{1}{ }^{4}}{384} \frac{d^{4} M_{0}}{d a^{4}}+\ldots
\end{aligned}
$$

${ }^{9}$ Electricity and Magnetism, Vol. II, Appendix II, Chapter XIV.

${ }^{10}$ Gray, Absolute Measurements, Vo1. II, Part II, p. 403. 
whence, $M_{1}+M_{3}-2 M_{0}=\frac{c_{1}^{2}}{4} \frac{d^{2} M_{0}}{d a^{2}}+\frac{c_{1}^{4}}{\mathrm{I} 9^{2}} \frac{d^{4} M_{0}}{d a^{4}}+\ldots$

Similarly, $M_{2}+M_{4}-2 M_{0}=\frac{b_{1}^{2}}{4} \frac{d^{2} M_{0}}{d x^{2}}+\frac{b_{1}{ }^{4}}{\mathrm{I}^{2}} \frac{d^{4} M_{0}}{d x^{4}}+\ldots$

In a similar manner, if $M_{5}, M_{6}, M_{7}, M_{8}$ are the coefficients of inductance of the single turn $\mathrm{O}_{1}$, on single turns at points $5,6,7,8$ of coil $B$, we get

$$
\begin{aligned}
& M_{5}+M_{7}-2 M_{0}=\frac{c_{2}^{2}}{4} \frac{d^{2} M_{0}}{d A^{2}}+\frac{c_{2}{ }^{4}}{\mathrm{I} 9^{2}} \frac{d^{4} M_{0}}{d A^{4}}+\ldots \\
& M_{6}+M_{8}-2 M_{0}=\frac{b_{2}^{2}}{4} \frac{d^{2} M_{0}}{d x^{2}}+\frac{b_{2}^{4}}{\mathrm{I} 9^{2}} \frac{d^{4} M_{0}}{d x^{4}}+\ldots
\end{aligned}
$$

Adding equations $(l)$ and $(m)$ we have

$$
\begin{aligned}
& \frac{\mathrm{I}}{6}\left(M_{1}+M_{2}+M_{3}+M_{4}+M_{5}+M_{6}+M_{7}+M_{8}-2 M_{0}\right) \\
& \quad=M_{0}+\frac{\mathrm{I}}{24}\left\{\left(b_{1}{ }^{2}+b_{2}{ }^{2}\right) \frac{d^{2} M_{0}}{d x^{2}}+c_{1} \frac{{ }^{2} M^{2}}{d a^{2}}+c_{2}{ }^{2} \frac{d^{2} M_{0}}{d A^{2}}\right\} \\
& \quad+\frac{\mathrm{I}}{\operatorname{II} 5^{2}}\left\{\left(b_{1}{ }^{4}+b_{2}{ }^{4}\right) \frac{d^{4} M_{0}}{d x^{4}}+c_{1}{ }^{4} \frac{d^{4} M_{0}}{d a^{4}}+c_{2}{ }^{4} \frac{d^{4} M_{0}}{d A^{4}}\right\}+\ldots
\end{aligned}
$$

The mutual inductance of two coils of unequal radii and unequal section is, neglecting sixth and higher differentials, ${ }^{11}$

$$
\begin{aligned}
M=M_{0} & +\frac{\mathrm{I}}{24}\left\{\left(b_{1}{ }^{2}+b_{2}{ }^{2}\right) \frac{d^{2} M_{0}}{d x^{2}}+c_{1}{ }^{2} \frac{d^{2} M_{0}}{d a^{2}}+c_{2}{ }^{2} \frac{d^{2} M_{0}}{d A^{2}}\right\} \\
& +\frac{\mathrm{I}}{\mathrm{I} 92 \mathrm{O}}\left\{\left(b_{1}{ }^{4}+b_{2}{ }^{4}\right) \frac{d^{4} M_{0}}{d x^{4}}+c_{1} \frac{{ }^{4} M_{0}}{d a^{4}}+c_{2}{ }^{4} \frac{d^{4} M_{0}}{d A^{4}}\right\} \\
& +\frac{\mathrm{I}}{576}\left\{b_{1}{ }^{2} b_{2}{ }^{2} \frac{d^{4} M_{0}}{d x^{4}}+c_{1}{ }^{2} c_{2}{ }^{2} \frac{d^{4} M_{0}}{d a^{2} d A^{2}}\right\} \\
& +\frac{\mathrm{I}}{576}\left(b_{1}{ }^{2}+b_{2}{ }^{2}\right)\left\{c_{1} \frac{a^{4} M_{0}}{d x^{2} d a^{2}}+c_{2}{ }^{2} \frac{d^{4} M_{0}}{d x^{2} d A^{2}}\right\}
\end{aligned}
$$

${ }^{11}$ Rosa, this Bulletin, p. 337 . 
For two equal coils this is

$$
\begin{aligned}
& M=M_{0}+\frac{\mathrm{I}}{\mathrm{I} 2}\left(b^{2} \frac{d^{2} M_{0}}{d x^{2}}+c^{2} \frac{d^{2} M_{0}}{d a^{2}}\right) \\
& +\frac{\mathrm{I}}{360}\left(b^{4} \frac{d^{4} M_{0}}{d x^{4}}+c^{4} \frac{d^{4} M_{0}}{d a^{4}}\right)+\frac{b^{2} c^{2}}{\mathrm{I} 44} \frac{d^{4} M_{0}}{d x^{2} d a^{2}}
\end{aligned}
$$

Equation (n) agrees with (I6) only when the fourth differentials are negligible. In that case we can write

$$
M=\frac{\mathrm{I}}{6}\left(M_{1}+M_{2}+M_{3}+M_{4}+M_{5}+M_{6}+M_{7}+M_{8}-2 M_{0}\right)
$$

For two coils of equal radii and equal section this becomes

$$
M=\frac{\mathrm{I}}{3}\left(M_{1}+M_{2}+M_{3}+M_{4}-M_{0}\right)
$$

Equation (I8) is Rayleigh's formula, or the formula of quadratures. Instead of computing the correction to $M_{0}$ by means of the differential coefficients (I3), eight additional values are computed, corresponding to the mutual inductances of the single turns at the eight points indicated in Fig. 4, each with reference to the central turn of the other coil. These $M s$ may be computed by formulæ (8) and (9) or (IO) and (II), and the values of the constants for the case of two coils of equal radii are given in the following table, the radius being $a$ in every case.

Axial distance.

Using (8)

“

“

“

Using (9)

6

16

"6
Radial distance.

$$
\begin{aligned}
& -\frac{c_{1}}{2} \\
& +\frac{c_{1}}{2} \\
& -\frac{c_{2}}{2} \\
& +\frac{c_{2}}{2}
\end{aligned}
$$

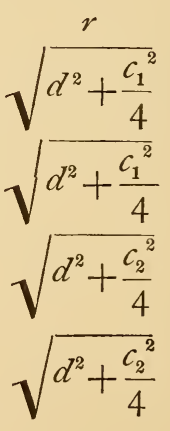

$d-b_{1} / 2 \quad 0$

$d+b_{1} / 2 \quad 0$

$d+b_{2} / 2 \quad 0$

$d-b_{2} / 2 \quad 0$ 
MAGNITUDE OF THE ERRORS IN ROWLAND'S AND RAYLEIGH'S FORIMLIE.

The error in equation (I8) is the difference between the values of $M$ as given by $(n)$ and (I6). Calling this correction $\epsilon_{1}$, and taking the difference for simplicity for the case of two equal coils,

$$
\epsilon_{1}=\frac{\mathrm{I}}{960}\left\{b^{4} \frac{d^{4} M_{0}}{d x^{4}}+c^{4} \frac{d^{4} M_{0}}{d a^{4}}\right\}+\frac{b^{2} c^{2}}{I 44} \frac{d^{4} M_{0}}{d x^{2} d a^{2}}
$$

The values of the differential coefficients of (I 7 ) are as follows: ${ }^{12}$

$$
\begin{aligned}
\frac{d^{4} M_{0}}{d x^{4}}=\frac{d^{4} M_{0}}{d \alpha^{4}} & =4 \pi a \times \frac{6}{d^{4}} \\
\frac{d^{4} M}{d x^{2} d a^{2}} & =-4 \pi a \times \frac{6}{d^{4}}
\end{aligned}
$$

Substituting these values in $(o)$ we have

$$
\epsilon_{1}=4 \pi a\left\{\frac{3 b^{4}+3 c^{4}-20 b^{2} c^{2}}{480 d^{4}}\right\}
$$

For a square coil the correction is a negative quantity, showing that $M$ by equation (I9) is too large, and the error is proportional to the fourth power of $\frac{I}{d}$, the reciprocal of the distance between the mean planes of the coils. For a rectangular coil in which $b$ is greater than $c$ the correction is negative so long as $b$ is not more than 2.5 times $c$. When $b$ is still larger with respect to $c$ the correction becomes plus, the value of $M$ by (I9) being too small.

Thus, for a coil of cross section 4 sq. $\mathrm{cm}$, we get the following values of the numerator of $(20)$ as we vary the shape of cross section, keeping $b c=4$.

$$
\begin{aligned}
& \text { Dimensions of coil. } \\
& b=2 \quad c=2 \\
& b=2.5 c=\mathrm{I} .6 \\
& b=3 \quad c=\mathrm{I} \cdot 33 \\
& b=4 \quad c=\mathrm{I} \\
& b=8 \quad c=0.5
\end{aligned}
$$

$$
\begin{aligned}
& \text { Error proportional to- } \\
& \begin{array}{c}
-\quad 224 \\
-\quad 183 \\
-\quad 67.5 \\
+\quad 45 \mathrm{I} \\
+\mathrm{II}, 988
\end{array}
\end{aligned}
$$

Thus we see that the value of $M$ as given by the formula of quadratures may be too large or too small according to the shape of the 
section, and that the error is proportional to the fourth power of the dimensions of the section divided by the distance between the mean planes of the coils. When the section is small and $d$ large the error will become negligible.

The error by Rowland's formula is found by taking the difference between (I4) and (I7). Thus the error $\epsilon_{2}$ is

$$
\epsilon_{2}=4 \pi a \frac{6}{d^{4}}\left\{\frac{b^{4}+c^{4}}{360}-\frac{b^{2} c^{2}}{144}\right\}=4 \pi a \frac{8 b^{4}+8 c^{4}-20 b^{2} c^{2}}{480 d^{4}}
$$

This is negative for a square coil, but smaller than $\epsilon_{1}$. For a coil of section such that $b=c \sqrt{2}$, this error is zero, and for sections such that that $\frac{b}{c}>\sqrt{2}$, the error is positive. Thus, for a coil of cross section 4 sq. $\mathrm{cm}$, we get the following values of the numerator of (2I) which is proportional to the error by Rowland's formula.

$$
\begin{array}{cl}
\text { Dimensions of coil. } \\
b=2 & c=2 \\
b=2.5 & c=\mathrm{I} .6 \\
b=3 & c=\mathrm{I} .33 \\
b=4 & c=\mathrm{I} \\
b=8 & c=0.5
\end{array}
$$

Error proportional to-

$\begin{array}{rr}- & 64 \\ + & 45 \\ + & 353 \\ + & 1,736 \\ + & 32,448\end{array}$

Thus the error is smaller by Rowland's formula for coils having square or nearly square section, but larger for coils having rectangular sections not nearly square.

These conclusions are verified by numerical calculations with the formulæ of Rowland and Rayleigh later in this paper.

\section{LYLE'S FORMULA.}

Professor Lyle ${ }^{13}$ has recently proposed a very convenient method for calculating the mutual inductance of coaxial coils, which gives very accurate results for coils at some distance from each other. Lyle begins his demonstration with Maxwell's ${ }^{14}$ expression for the magnetic potential of a coil at any point in its axis, namely,

$$
V=2 \pi n C\left\{I-\frac{x}{\rho}+\frac{c^{2} x}{24 \rho^{3}}+\frac{b^{2}-c^{2}}{8 \rho^{5}} \cdot a^{2} x\right\}
$$

\footnotetext{
${ }^{13}$ Phil. Mag., 3, p. 310; 1902. ${ }^{14}$ Electricity and Magnetism, Vo1. II, \& 700.
} 
assuming the dimensions of the cross section small in comparison with the radius of the coil, and the winding uniform.

In the above equation,

$$
\begin{aligned}
& a=\text { mean radius of the coil } \\
& b=\text { axial breadth, } \\
& c=\text { radial depth, } \\
& x=\text { distance on axis from center of coil, } \\
& \rho=\sqrt{a^{2}+x^{2}}
\end{aligned}
$$

$C$ is the current, and $n$ is the number of turns in the coil. This

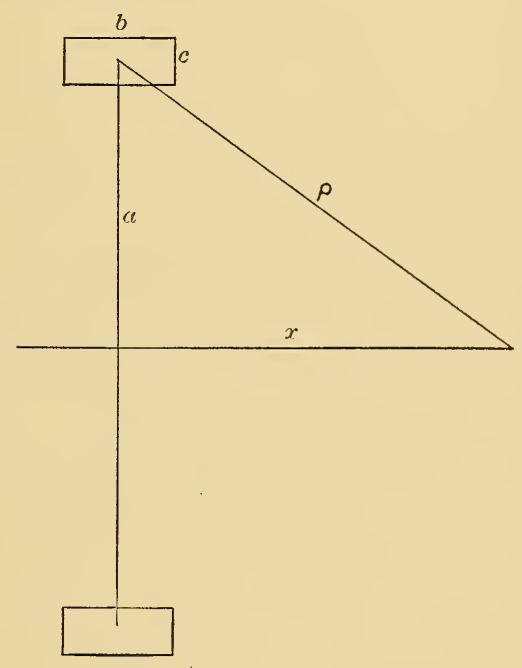

Fig. 5

notation differs from Lyle's only in using, as elsewhere in this article, $b$ and $c$ for the breadth and depth of coil, instead of $\xi$ and $\eta$.

Expanding the above equation in ascending powers of $\frac{x}{a}$, Lyle obtains,

$$
\begin{aligned}
V=2 \pi n C & {\left[\mathrm{I}-\frac{x}{a}\left(\mathrm{I}-\frac{3 b^{2}-2 c^{2}}{24 a^{2}}\right)+\frac{x^{3}}{2 a^{3}}\left(\mathrm{I}-\frac{3\left(5 b^{2}-4 c^{2}\right)}{24 a^{2}}\right)\right.} \\
& \left.-\frac{3 x^{5}}{2 \cdot 4 a^{5}}\left(\mathrm{I}-\frac{5\left(7 b^{2}-6 c^{2}\right)}{24 a^{2}}\right)+\frac{3 \cdot 5 x^{7}}{2 \cdot 4 \cdot 6 a^{2}}\left(\mathrm{I}-\frac{7\left(9 b^{2}-8 c^{2}\right)}{24 a^{2}}\right)-.\right]
\end{aligned}
$$


The potential $V^{\prime}$ of a circular filament of radius $r$ and carrying current $n C$ at a point $\mathrm{P}$ distant $x$ from the center of the circle is

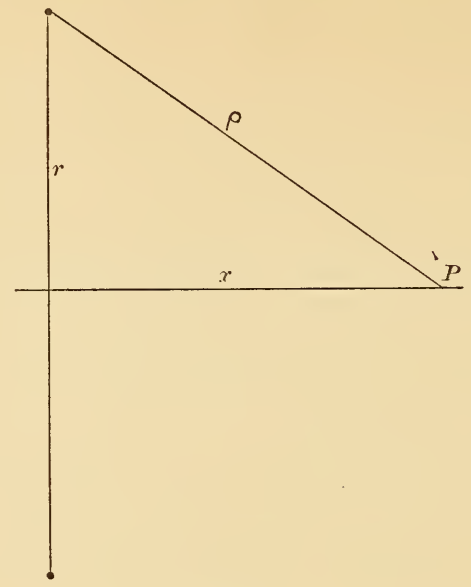

Fig. 6

$$
V^{\prime}=2 \pi n C\left\{\mathrm{I}-\frac{x}{\sqrt{x^{2}+r^{2}}}\right\}
$$

which expanded in ascending powers of $\frac{x}{r}$ gives

$$
V^{\prime}=2 \pi n C\left\{\mathrm{I}-\frac{x}{r}+\frac{x^{3}}{2 r^{3}}-\frac{\mathrm{I} \cdot 3 x^{5}}{2 \cdot 4 r^{5}}+\frac{\mathrm{I} \cdot 3 \cdot 5 x^{7}}{2 \cdot 4 \cdot 6 r^{7}}-\ldots\right\}
$$

The two potentials $V$ in $(q)$ and $V^{\prime}$ in $(s)$ will be identical, provided

$$
\begin{aligned}
& \frac{\mathrm{I}}{r}=\frac{\mathrm{I}}{a}\left\{\mathrm{I}-\frac{3 b^{2}-2 c^{2}}{24 a^{2}}\right. \\
& \frac{\mathrm{I}}{r^{3}}=\frac{\mathrm{I}}{a^{3}}\left\{\mathrm{I}-\frac{3\left(5 b^{2}-4 c^{2}\right)}{24 a^{2}}\right\} \\
& \frac{\mathrm{I}}{r^{5}}=\frac{\mathrm{I}}{a^{5}}\left\{\mathrm{I}-\frac{5\left(7 b^{2}-6 c^{2}\right)}{24 a^{2}}\right\}
\end{aligned}
$$

If the section is square, and hence $b=c$, these equations become

$$
\begin{aligned}
& \frac{\mathrm{I}}{r}=\frac{\mathrm{I}}{a}\left(\mathrm{I}-\frac{b^{2}}{24 a^{2}}\right) \\
& \frac{\mathrm{I}}{r^{3}}=\frac{\mathrm{I}}{a^{3}}\left(\mathrm{I}-\frac{3 b^{2}}{24 a^{2}}\right) \\
& \frac{\mathrm{I}}{r^{5}}=\frac{\mathrm{I}}{a^{5}}\left(\mathrm{I}-\frac{5 b^{2}}{24 a^{2}}\right)
\end{aligned}
$$


Cubing the first of these equations we have

$$
\frac{\mathrm{I}}{r^{3}}=\frac{\mathrm{I}}{a^{3}}\left(\mathrm{I}-\frac{3 b^{2}}{24 a^{2}}+\frac{3 b^{4}}{24^{2} a^{4}}-\frac{b^{6}}{24^{3} a^{6}}\right)
$$

Hence, if we neglect the terms in $\frac{b^{4}}{a^{4}}$ and higher powers of $\left(\frac{b}{a}\right)$ we see that all the equations of condition $(t)$ are satisfied by making $b=c$. The same result follows if the expansions are in ascending powers of $\frac{a}{x}$.

The first of equations $(u)$ gives

$$
r=a\left(\mathrm{I}+\frac{b^{2}}{24 a^{2}}\right)
$$

again neglecting fourth and higher powers of $\frac{b}{a}$. Hence, we see that if a coil of radius $a$, cross section $b^{2}$, wound with $n$ turns of wire and carrying a current $C$, be replaced by a single filament lying in the mean plane of the coil, of radius $r$ and carrying a current $n C$, the magnetic potential will be identical at all points on the axis, and hence, by Legendre's theorem, identical at all points of space without the coil. Thus, this filament is seen to be equivalent to the coil and can replace it so far as its external field is concerned. If there were $n$ turns in the filament $\mathrm{O}$, through which current $C$ flows, the flux due to $\mathrm{O}$ through coil $B$ would be the same as that due to current $C$ through the $n$ turns of A.

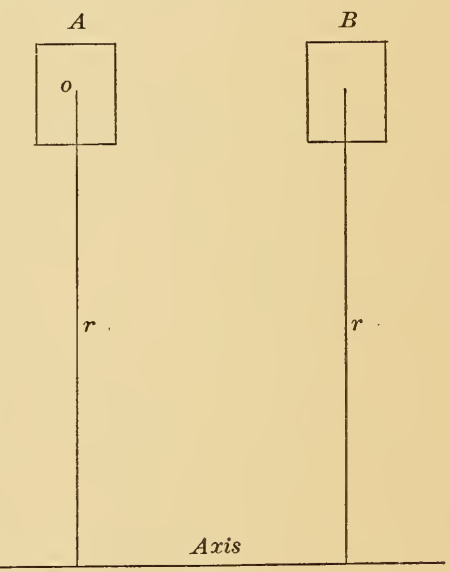

Fig. 7

But since the mutual inductance is the same whichever coil is the primary, the flux through $\mathrm{O}$ due to current $C$ in $\mathrm{B}$ must be the same as it is through the $n$ turns of $\mathrm{A}$, and therefore the filament O can replace the coil A, not only so far as its own external field is concerned, but also so far as the effect of external fields on it is concerned. 
In the proof of equation $(p)$, however, differential coefficients of the fourth and higher orders have been neglected, and hence when we apply Legendre's theorem and pass to space off the axis we must keep away from the immediate region of the coil itself, where the fonth and higher differentials of the magnetic potential can not be neglected. Obviously a single filament can not replace a coil of rectangular section just outside the coil, and we shall see later that it does so to a high order of approximation only at some distance from it.

Lyle then goes on to show that a coil of rectangular section not square can be replaced by two filaments, the distance apart of the filaments being called the equivalent breadth or the equivalent depth of the coil.

$$
\left.\begin{array}{l}
\beta^{2}=\frac{b^{2}-c^{2}}{\mathrm{I} 2}, 2 \beta \text { is the equivalent breadth of } \mathrm{A} \\
\delta^{2}=\frac{c^{2}-b^{2}}{\mathrm{I} 2}, 2 \delta \text { is the equivalent depth of } \mathrm{B}
\end{array}\right\}
$$

The equivalent radius of $\mathrm{A}$ is given by the same expression which holds for a square coil, viz:

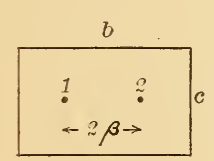

$A$

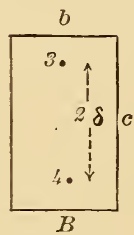

Fig. 8

$$
r=a\left(\mathrm{I}+\frac{c^{2}}{24 a^{2}}\right)
$$

In the coil $\mathrm{B}$ the equivalent filaments have radii $r+\delta$ and $r-\delta$, respectively, where

$$
r=\alpha\left(\mathrm{I}+\frac{32}{24 \alpha^{2}}\right)
$$

The inutual inductance of two coils may now be readily calculated. If each has a square section, it is necessary only to calculate the mutual inductance of the two equivalent filaments. For coils of rectangular sections, as $\mathrm{A}, \mathrm{B}$, the mutual inductance will be the sum of the mutual inductances of the two filaments of $\mathrm{A}$ on the two filaments of $\mathrm{B}$, counting $n / 2$ turns in each. Or, it is $n_{1} n_{2}$ times the mean of the four inductances $M_{13}, M_{14}, M_{23}, M_{24}$, where $M_{13}$ is the mutual inductance of filament I on filament 3 , etc. 
Similarly the attractions of coils when carrying currents may be calculated.

In a uniform magnetic field the equivalent radius of a coil is easily found as follows. The mutual inductance of two coils is proportional to the whole number of lines of force due to A linked with the various turns of coil B. For a uniform field this is proportional to the sum of the areas of the various turns of the coil. We can therefore find the equivalent radius $r_{0}$ for a coil of rectangular section by integration, $r_{1}$ and $r_{2}$ being the inner and outer radii of the coil. Thus-

$$
\begin{aligned}
& \pi r_{0}^{2}=\frac{\pi}{r_{2}-r_{1}} \int_{r_{1}}^{r_{2}} r^{2} d r=\frac{\pi}{r_{2}-r_{1}}\left[\frac{r_{2}^{3}-r_{1}^{3}}{3}\right] \\
& \therefore r_{0}^{2}=\frac{\mathrm{I}}{3}\left(r_{1}^{2}+r_{1} r_{2}+r_{2}^{2}\right)=\left(\frac{r_{1}+r_{2}}{2}\right)\left[\mathrm{I}+\frac{\mathrm{I}}{3}\left(\frac{r_{2}-r_{1}}{r_{2}+r_{1}}\right)^{2}\right]
\end{aligned}
$$

If $\alpha$ is the mean radius of the coil and $c$ is the radial depth

$$
\begin{aligned}
a & =\frac{r_{1}+r_{2}}{2} \\
c & =r_{2}-r_{1} \\
\therefore r_{0}^{2} & =a^{2}\left(\mathrm{I}+\frac{c^{2}}{\mathrm{I} 2 a^{2}}\right) \\
\text { or } r_{0} & =a\left(\mathrm{I}+\frac{c^{2}}{24 a^{2}}\right)
\end{aligned}
$$

neglecting terms in the fourth and higher powers of $\left(\frac{c}{a}\right)$. This value of the equivalent radius which applies to any coil of rectangular section in a uniform field is exactly the value found above for a coil of square section in a non-uniform field, where fourth and higher differentials are negligible.

Lyle states that in his method of obtaining the mutual inductance no quantities are neglected of order lower than the fourth in $\left(\frac{b}{a}\right)$. It is to be observed, however, that that statement only applies when the coils are a considerable distance apart, as the tern neglected depend- 
ing on the fourth differentials is proportional to $\left(\frac{b}{d}\right)^{4}$, and this may be much larger than $\left(\frac{b}{a}\right)^{4}$.

Lyle's method is of special value in computing mutual inductances because it applies to coils of nnequal as well as of equal radii. Examples and tests of the method will be given later.

We will now deduce an expression for $\Delta M$ based on Lyle's value of the equivalent radius (22). Thus, putting $a_{1}$ for the equivalent radius where $a$ is the mean radius, we have as before

$$
a_{1}=a\left(\mathrm{I}+\frac{b^{2}}{24 \alpha^{2}}\right)
$$

We may use this value of the radius in any formula for the mutual inductance of two coaxial circles, as I, 2, 5, or 9. Substituting in (9) we have for $M$ and $M_{0}$,

$$
\begin{aligned}
& M=4 \pi a_{1}\left\{\log \frac{8 a_{1}}{d}\left(\mathrm{I}+\frac{3}{\mathrm{I} 6} \frac{d^{2}}{a_{1}^{2}}-\ldots\right)-\left(2+\frac{d^{2}}{\mathrm{I} 6 a_{1}{ }^{2}}-\ldots\right)\right\} \\
& M_{0}=4 \pi a\left\{\log \frac{8 a}{d}\left(\mathrm{I}+\frac{3}{\mathrm{I} 6} \frac{d^{2}}{a^{2}}-\ldots\right)-\left(2+\frac{d^{2}}{\mathrm{I} 6 a^{2}}-\ldots\right)\right\}
\end{aligned}
$$

The correction $\Delta M$ is found by taking the difference between $M$ and $M_{0}$. Thus,

$$
\begin{aligned}
& \Delta M=4 \pi\left(a_{1}-a\right)\left\{\log \frac{8 a}{d}\left(\mathrm{I}+\frac{3}{\mathrm{I} 6} \frac{d^{2}}{a^{2}}\right)-\left(2+\frac{d^{2}}{\mathrm{I} 6 a^{2}}\right)\right\} \\
& +4 \pi a\left\{\log \frac{a_{1}}{a}\left(\mathrm{I}+\frac{3}{\mathrm{I} 6} \frac{d^{2}}{a^{2}}\right)-\log \frac{8 a}{d}\left(\frac{3 d^{2}}{\mathrm{I} 6} \cdot \frac{a_{1}{ }^{2}-a^{2}}{a_{4}}\right)+\frac{d^{2}}{\mathrm{I} 6} \cdot \frac{a_{1}{ }^{2}-a^{2}}{a_{4}}\right\} \\
& =4 \pi a\left\{\frac{b^{2}}{24 a}\left[\left(\log \frac{8 a}{d}-2\right)+\frac{3 d^{2}}{16 a^{2}} \log \frac{8 a}{d}-\frac{d^{2}}{16 a^{2}}\right]\right. \\
& \left.+\frac{b^{2}}{24 a^{2}}+\frac{b^{2} d^{2}}{\mathrm{I} 28 a^{4}}-\frac{3 b^{2} d^{2}}{\mathrm{I} 92 a^{4}} \log \frac{8 a}{d}+\frac{b^{2} d^{2}}{\mathrm{I} 92 a^{4}}\right\} \\
& \text { Since } \quad \frac{a_{1}-a}{a}=\frac{b^{2}}{24 a^{2}} \text { by assumption, } \\
& a_{1}{ }^{2}-a^{2}=\frac{b^{2}}{\mathrm{I} 2}, \text { approximately, }
\end{aligned}
$$




$$
\text { and } \log \frac{a_{1}}{a}=\frac{b^{2}}{24 \alpha^{2}}, \text { approximately. }
$$

Combining terms in $(v)$

$$
\begin{gathered}
\Delta M=4 \pi a\left\{\frac{b^{2}}{24 a^{2}}\left(\log \frac{8 a}{d}-\mathrm{I}\right)-\frac{b^{2} d^{2}}{\mathrm{I} 28 a^{4}}\left(\log \frac{8 a}{d}-\frac{4}{3}\right)\right\} \\
\frac{\Delta M}{\pi}=\frac{b^{2}}{6 a}\left\{\log \frac{8 a}{d}-\mathrm{I}-\frac{3 d^{2}}{\mathrm{I} 6 a^{2}}\left(\log \frac{8 a}{d}-\frac{4}{3}\right)\right\}
\end{gathered}
$$

Comparing this equation with $(27)$ we see that it differs only in the absence of the two terms $-\frac{a^{2} b^{2}}{5 d^{4}}$ and $+\frac{17 b^{2}}{240 d^{2}}$. These terms depend on the fourth differentials, which, as stated above, were ignored in deriving equation $(p)$. Thus we see that, if in deriving equation (27) we had ignored fourth differentials, we should have come to the same result that Lyle has, although the process is very different and the form of the result is very different. Since Rowland's formula depends on second differentials only, we should expect it to agree closely with Lyle's, and we shall see presently that for coils of square section it does.

In a similar manner we may obtain an expression for $\Delta M$ for two coils of unequal radii. Substituting $a_{1}$, the equivalent radius in (8), and putting $y$ and $y_{1}$ for $c$, we have:

$$
\begin{aligned}
M=4 \pi & a_{1}\left\{\log \frac{8 a_{1}}{r_{1}}\left(\mathrm{I}+\frac{y_{1}}{2 a_{1}}+\frac{y_{1}{ }^{2}+3 d^{2}}{\mathrm{I} 6 a_{1}{ }^{2}}-\frac{y_{1}{ }^{3}+3 v_{1} d^{2}}{32 a_{1}^{3}}-\ldots\right)\right. \\
- & \left.\left(2+\frac{y_{1}}{2 a}-\frac{3 y_{1}{ }^{2}-d^{2}}{\mathrm{I} 6 a_{1}{ }^{2}}+\frac{y_{1}{ }^{3}-6 y_{1} d^{2}}{48 a_{1}{ }^{3}}-\ldots\right)\right\} \\
M_{0}=4 \pi a & \left\{\log \frac{8 a}{r}\left(\mathrm{I}+\frac{y}{2 a}+\frac{y^{2}+3 d^{2}}{\mathrm{I} 6 a^{2}}-\frac{y^{3}+3 y d^{2}}{32 a^{3}}-\cdots\right)\right. \\
- & \left.\left(2+\frac{y}{2 a}-\frac{3 y^{2}-d^{2}}{\mathrm{I} 6 a^{2}}+\frac{y^{3}-6 y d^{2}}{48 a^{3}}\right)\right\}
\end{aligned}
$$

Putting as before the difference between these two expressions equal to $\Delta M$, we have:

$$
\frac{\Delta M}{\pi}=\frac{b^{2}}{6 a}\left(\log \frac{8 a}{r}+\frac{a y^{2}}{A r^{2}}\right)\left(\mathrm{I}+\frac{y}{2 a}+\frac{y^{2}+3 d^{2}}{\mathrm{I} 6 a^{2}}-\frac{y^{3}+3 y d}{32 a^{3}}\right)
$$

$$
\text { 16360-07-5 }
$$




$$
\begin{aligned}
& -\mathrm{I}+\frac{2 y^{2}+d^{2}}{8 a^{2}}-\frac{5 y^{3}-3 y d^{2}}{96 a^{3}}-\frac{(A+a) y}{2 A a}\left(\log \frac{8 a}{r}-\mathrm{I}\right) \\
& -\frac{(A+a) y^{2}}{8 A a^{2}}\left(\log \frac{8 a}{r}+3\right)-\frac{3 d^{2}}{8 a^{2}}\left(\log \frac{8 a}{r}-\frac{\mathrm{I}}{3}\right) \\
& \left.+\frac{3(A+a) y^{3}}{32 A a^{3}}\left(\log \frac{8 a}{r}+\frac{2}{3}\right)+\begin{array}{c}
3(3 A+a) d^{2} y\left(\log \frac{8 a}{r}-\frac{4}{3}\right) \\
32 A a^{3}
\end{array}\right\}
\end{aligned}
$$

This applies to coils of equal square sections, of radii $a$ and $A$, distance between centers being $d ; y=A-a$.

This formula is easier to use than it might appear to be. There is only one natural logarithm to get, and when one is calculating $\Delta M$ directly it is not necessary to work to so high precision as when calculating $M$. If, however, one wishes only $M$ and not $\Delta M$ it would be better to calculate it directly by Lyle's method.

\section{STEFAN'S FORIIULA}

Stefan's ${ }^{15}$ formula for the mutual inductance of two equal coaxial coils is as follows:

$$
\begin{aligned}
& M=4 \pi a n^{2}\left\{\log _{\frac{8}{d}} \frac{8 a}{2}+\frac{b^{2}-c^{2}}{\mathrm{I} 2 d^{2}}+\frac{2 b^{4}+2 c^{4}-5 b^{2} c^{2}}{\mathrm{I} 20 d^{4}}+\frac{3 b^{6}-7 b^{4} c^{2}+7 b^{2} c^{4}-3 c^{6}}{504 d^{6}}\right. \\
& \left.+\left(\log \frac{8 a}{d}-2\right)\left(\frac{3 b^{2}+c^{2}+\mathrm{I} 8 d^{2}}{96 a^{2}}-\frac{\mathrm{I} 5 d^{4}}{\mathrm{IO} 24 a^{4}}\right)+\frac{7 b^{2}+23 c^{2}+60 d^{2}}{192 a^{2}}-\frac{29 d^{4}}{2048 a^{4}}\right\}
\end{aligned}
$$

This formula ${ }^{16}$ may be written $M=M_{0}+\Delta M$ where $M_{0}$ is the mutual inductance of the central circles of the two coils and $\Delta M$ is the correction for the section of the coil, but the value of $\lrcorner M$ in formula (25) is incorrect. The corrected expression for $J M$ is as follows : ${ }^{16}$

$$
\begin{gathered}
\frac{\Delta M}{\pi n^{2}}=4 a\left\{\frac{3 b^{2}+c^{2}}{96 a^{2}} \cdot \log \frac{8 a}{d}-\frac{\mathrm{II} b^{2}-3 c^{2}}{\mathrm{I} 92 a^{2}}+\frac{b^{2}-c^{2}}{\mathrm{I} 2 d^{2}}+\frac{2 b^{4}+2 c^{4}-5 b^{2} c^{2}}{\mathrm{I} 20 d^{4}}\right. \\
+\frac{6 b^{4}+6 c^{4}+5 b^{2} c^{2}}{5760 a^{2} d^{4}}+\frac{3 b^{6}-3 c^{6}+\mathrm{I} 4 b^{2} c^{4}-\mathrm{I} 4 b^{4} c^{2}}{504 d^{6}}+\frac{7 c^{2} d^{2}}{\mathrm{IO} 24 a^{4}}\left(\log \frac{8 a}{d}-\frac{\mathrm{I} 63}{84}\right) \\
\left.-\frac{15 b^{2} d^{2}}{\mathrm{I0} 24 a^{4}}\left(\log \frac{8 a}{d}-\frac{97}{60}\right)\right\}
\end{gathered}
$$

\footnotetext{
${ }^{15}$ Wied. Annalen, 22, p. 107; I884. ${ }^{16}$ Rosa, this Bulletin, p. $348,(38)$ and (39).
} 
For a square section, when $b=c$, this becomes

$$
\frac{\Delta M}{\pi n^{2}}=\frac{b^{2}}{6 a}\left\{\log \frac{8 a}{d}-\mathrm{I}-\frac{a^{2} b^{2}}{5 d^{4}}-\frac{3 d^{2}}{16 a^{2}}\left(\log \frac{8 a}{d}-\frac{4}{3}\right)+\frac{\mathrm{I} 7 b^{2}}{240 d^{2}}\right\}
$$

The last two terms of equation (27) are resatively small, so that we may write, approximately:

$$
\frac{4 M}{\pi n^{2}}=\frac{b^{2}}{6 a}\left\{\log \frac{8 a}{d}-\mathrm{x}-\frac{a^{2} b^{2}}{5 d^{4}}\right\}
$$

These expressions for $\Delta M$ are very exact where the coils are near together or where they are separated for a considerable distance, but become less exact as $d$ is greater. They are therefore most reliable where formulæ (I4), (I9), and (22) are least reliable. As formula $(28)$ is exact enough for most purposes, it affords a very easy method of getting the correction for equal coils of square section.

We give later examples to illustrate and test the accuracy of the above formulæ.

\section{WEINSTEIN'S FORMULA.}

Weinstein ${ }^{17}$ gives a formula for the mutual inductance of equal coaxial coils, as follows:

$$
\begin{aligned}
M=C & \left\{(K-E)\left[\epsilon+\frac{\cos ^{2} \lambda}{\mathrm{I} 2 h^{2}}\left(a_{1}-a_{2}-a_{3}+\left(2 a_{2}-3 a_{3}\right) \cos ^{2} \lambda+8 a_{3} \cos ^{4} \lambda\right)\right]\right. \\
& -E\left[\mathrm{I}-\frac{\sin ^{2} \lambda}{\mathrm{I} 2 h^{2}}\left(a_{1}+\frac{a_{2}}{2}+2 a_{3}+\left(2 a_{2}+3 a_{3}\right) \cos ^{2} \lambda+8 a_{3} \cos ^{4} \lambda\right)\right](29)
\end{aligned}
$$

where $C=2 \pi d n_{1} n_{2} \sin \lambda, d$ is the mean diameter of the coil, and $K$ and $E$ are elliptic integrals. The above expression also is of the form $M=M_{0}+\Delta M$, and can be used better in the second form, where $M_{0}$ can be calculated by any one of several reliable formulæ and $\Delta M$ can be computed separately. The expression for $\Delta M$ derived from (29) is as follows: $:^{18}$

$$
\frac{\Delta H}{\pi n_{1} n_{2}}=4 a \sin \gamma\left\{(F-E)\left(A+\frac{c^{2}}{24 a^{2}}\right)+E B\right\}
$$

\footnotetext{
${ }^{17}$ Wied. Annalen, 21, p. 350; ISS4. $\quad$ 1n Rosa, this Bulletin, p. 342, (20).
} 
where $F$ and $E$ are the complete elliptic integrals to modulus $\sin \gamma$, (as in equation $x$ ) and

$$
\begin{aligned}
& A=\frac{\cos ^{2} \gamma}{\mathrm{I} 2 d^{2}}\left(a_{1}-a_{2}-a_{3}+\left(2 a_{2}-3 a_{3}\right) \cos ^{2} \gamma+8 a_{3} \cos ^{4} \gamma\right) \\
& B=\frac{\sin ^{2} \gamma}{\mathrm{I} 2 d^{2}}\left(a_{1}+\frac{a_{2}}{2}+2 a_{3}+\left(2 a_{2}+3 a_{3}\right) \cos ^{2} \gamma+8 a_{3} \cos ^{4} \gamma\right)
\end{aligned}
$$

The values of $a_{1}, a_{2}$, and $a_{3}$ are as follows:

$$
\begin{aligned}
& a_{1}=b^{2}-c^{2}+\frac{c^{4}}{30 a^{2}} \quad \text { For square section: } a_{1}=\frac{b^{4}}{30 a^{2}} \\
& a_{2}=\frac{5 b^{2} c^{2}-4 c^{4}}{60 a^{2}} \quad \text { " } \quad \text { " } \quad a_{2}=\frac{b^{4}}{60 a^{2}} \\
& a_{3}=\frac{2 b^{4}+2 c^{4}-5 b^{2} c^{2}}{20 d^{2}} \quad \text { " } \quad \text { " " } \quad a_{3}=-\frac{b^{4}}{20 d^{2}}
\end{aligned}
$$

This formula (30) is a very exact formula for all positions of the two coils, except when they are quite close together. We give later illustrations and tests of the formula and a comparison of its results with the results of other formulæ.

\section{USE OF FORMULE FOR SELF-INDUCTANCE IN CALCULATING MUTUAL INDUCTANCE.}

One can obtain the mutual inductance of adjacent coils, or of coils at a distance from one another, by means of a formula for the selfinductance of coils. Thus, suppose we have a coil of rectangular section, which we subdivide into three equal

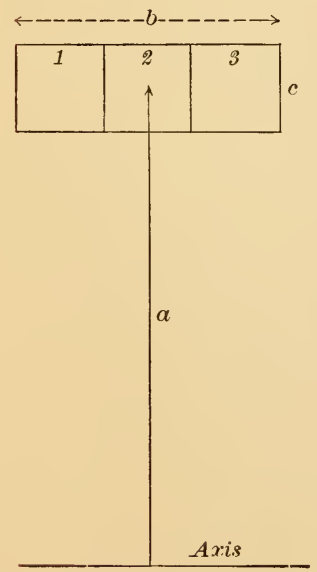

Fig. 9 parts, I, 2, 3, Fig. 9. Let $L$ be the self-inductance of the whole coil, $L_{1}$ be the self-inductance of any one of the three equal smaller coils, and $L_{2}$ be the self-inductance of two adjacent coils taken together. Also let $M_{12}$ be the mutual inductance of coil I on coil 2 , or of coil 2 on coil 3 , and $M_{13}$ be the mutual inductance of coil I on coil 3. Then,

$$
\left.\begin{array}{rl}
L & =3 L_{1}+4 M_{12}+M_{13} \\
\text { Also, } L_{2} & =2 L_{1}+2 M_{12} \\
\therefore M_{12} & =\frac{L_{2}-2 L_{1}}{2} \\
M_{13} & =\frac{L+L_{1}-2 L_{2}}{2}
\end{array}\right\}
$$


Formula (3I) will thus enable us to find the mutual inductance of two coils of equal radii adjacent or near each other by the calculation of self-inductances from such formulæ as those of Weinstein and Stefan. These latter formulæ are not, however, exact enough when the section is large to permit us to apply it to coils at any considerable distance from one another.

\section{GEOMETRIC MEAN DISTANCE FORIULA.}

The mutual inductance of two coaxial coils adjacent or very near can sometimes be obtained by means of the geometric mean distances. This method is accurate only when the sections are very small relatively to the radius. It can often be used to advantage in testing other formulæ, but not often in determining the mutual inductance of actual coils.

Formula (8) gives the mutual inductance of two very near coaxial coils in terms of the geometric mean distance, if $r$ be replaced by $R$, the geometric mean distance of the two sections. Formula (8) gives $M_{0}$ if $r$ be used, where $r$ is the distance between centers. Thus,

$$
\frac{\Delta M}{\pi n^{2}}=4 a \cdot \log \frac{r}{R} \cdot\left(\mathrm{I}+\frac{c}{2 a}\right)
$$

For coils $\mathrm{A}$ and $\mathrm{C}, R<r$ and $\Delta M$ is positive. " " A " $\mathrm{B}, R>r$ and $\Delta M$ is negative.

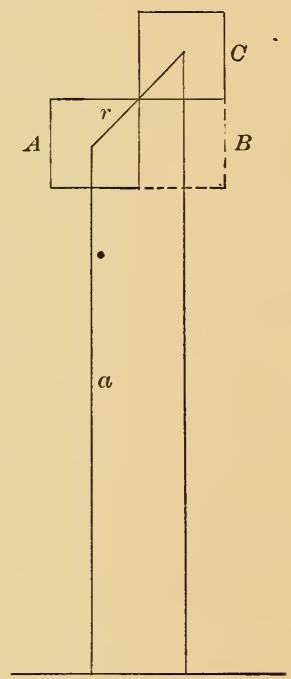

Fig. 10

\section{FORMULA AND EXAMPLES.}

\section{FORMULAE FOR CIRCLES.}

I. Maxwell.

2. Maxwell.
Absolute, in elliptic integrals, for all cases. Absolute (derived from I by Landen's transformation), especially for circles near together.

Series for $F$, complete elliptic integral of first kind. 
5. Weinstein.

6. Nagaoka.

7. Nagaoka.

8. Naxwell.

9. Maxwell.

IO. Coffin.

I I.

I 2.
Series for $E$, complete elliptic integral of second kind.

These formulæ are especially convenient for circles close together.

Series for $M$, using complementary modulus $k^{\prime} ; d$ small.

Using $q$-series and $k^{\prime} ; d$ large.

Using $q$-series and $k ; d$ small.

Algebraic series, $d$ small, radii nnequal.

Algebraic series, $d$ small, radii equal.

Fxtension of (9), radii equal.

Extension of (8), radii unequal.

For coplanar coaxial circles.

FORMUL E FOR COILS OF RECTANGULAR SECTION.

I3. Rowland.

I4. Rowland.

I 5 .

I6.

I 7 .

I8. Rayleigh.

I9. Rayleigh.

$2 \mathrm{O}$.

$2 \mathrm{I}$.

22. Lyle.

23. Lyle.

24 .
Coils of equal radii but unequal section.

Coils of equal radii and equal section.

Expressionis for $\frac{d^{2} M_{0}}{d x^{2}}$ and $\frac{d^{2} M_{0}}{d a^{2}}$.

Differential equation for mutual inductance of two coaxial coils.

Differential equation for mutual inductance of two coaxial coils of equal section and equal radii.

Formula of quadratures. $\quad M$ as mean of 9 values. (Radii may be unequal, but distance must not be sinall.)

Same for coils of equal radii and equal section.

Expression for error of Rayleigh's formula. Expression for error of Rowland's formula. Using an equivalent radius, square section. (Radii may be unequal, but distance must not be too small.)

Same for coils of sections not square.

Expression for $\Delta M$ based on Lyle's theorem, radii equal; sections square and equal. 
$24 a$.

25. Stefan.

26. Rosa.

27. Rosa.

28. Rosa.

29. Weinstein.
Expression for $\Delta M$ based on Lyle's theorem, radii unequal; sections square and equal. Algebraic series for $M$, radii equal. (Error in formula.)

Formula for $\Delta M$, radii equal, rectangular section.

Formula for $\Delta M$, radii equal, square section.

Formula for $\Delta M$, radii equal, simple approximate formula, square section.

Formula for $M$, using elliptic integrals. (Small error in formula.)

30. Rosa-Weinstein. Revised formula, expression for $\Delta M$.

$3 \mathrm{I}$.

Formula for $M$ in terms of self-inductances.

32.
Formula for $M$ by geometric mean distance.

\section{ILLUSTRATION AND TESTS OF THE FORMULA.}

\section{COAXIAL CIRCLES.}

Formula I.-Maxwell's. For any two coaxial circles.

Example I: Let $a=A=25 \mathrm{~cm}$ $d=20 \mathrm{~cm}$.

$$
\begin{aligned}
& k=\frac{50}{\sqrt{2500+400}}=0.9284766=\sin \gamma \\
& \gamma=68^{\circ} \text { I I }^{\prime} 54^{\prime \prime} 88=68^{\circ}{ }^{\prime}{ }^{\prime} 9^{8} 57^{8} .
\end{aligned}
$$

From Legendre's tables, we obtain

$\log F=0.385219 \mathrm{I}$

$\log E=0.0547850$

$$
\begin{aligned}
\frac{2}{k}-k=1.2255^{892} \quad\left(\frac{2}{k}-k\right) F & =2.975528 \mathrm{I} \\
\frac{2}{k}=2.1540658, \quad-\frac{2}{k} E & =\underline{-2.443678 \mathrm{I}} \\
\left(\frac{2}{k}-k\right) F-\frac{2}{k} E & =0.5318500 \\
4 a & =100 \\
\therefore \frac{M}{\pi} & =53.1850 \mathrm{~cm}
\end{aligned}
$$


Using Maxwell's tables (given in Maxwell, Appendix II, Vol. II)

$$
\begin{aligned}
\log _{10} \frac{M}{4 \pi a} \text { for } 68^{\circ} \mathrm{I} & =\overline{\mathrm{I}} .7230640 \\
\text { for } 68.2 & =\mathrm{I} .725^{\circ} 8286 \\
\text { for } 68^{\circ} \mathrm{I} 98578 & =\mathrm{I} .7257893 \text { by simple interpolation. } \\
\therefore \frac{M}{4 \pi a} & =0.531850 \\
\frac{M}{\pi} & =53.1850 \mathrm{~cm}, \text { as above. }
\end{aligned}
$$

The calculation of mutual inductance by the above methods is simplest for circles not near each other, as then the values of $\log F$, $\log E$, and $\log \frac{M}{4 \pi \sqrt{A a}}$ are very exact when taken by simple interpolation. When $\gamma$ is nearly $90^{\circ}$, however, second and third differences have to be used in interpolation.

Formula 2.-Maxwell's second expression. For circles near each other.

$$
\text { Exainple 2: } \begin{aligned}
\text { Let } a & =A=25 \mathrm{~cm} \\
d & =\mathrm{I} \mathrm{cm}
\end{aligned}
$$

In this case $k=\sin \gamma=\frac{50}{\sqrt{2501}}=.99980006$

$$
\gamma=88^{\circ} \quad 5^{\prime} \quad \mathrm{I}^{\prime \prime}
$$

This value of $\gamma$ is so nearly $90^{\circ}$ that it is difficult to obtain accurate values of $F$ and $E$ from tables of elliptic integrals, or of $\frac{M}{4 \pi a}$ from Maxwell's table.

We may therefore use formula (2) instead of (I).

$$
\begin{array}{r}
r_{1}=\sqrt{25 \mathrm{OI}}=5 \text { O.OI nearly, } r_{2}=\mathrm{I} . \mathrm{O} \\
\therefore k_{1}=\sin \gamma_{1}=\frac{r_{1}-r_{2}}{r_{1}+r_{2}}=\frac{49 . \mathrm{OI}}{5 \mathrm{I} . \mathrm{OI}}=0.960792 \\
\gamma_{1}=73^{\circ} 54^{\prime} 9 .^{\prime \prime} 7=73^{\circ} \cdot 9027
\end{array}
$$


From Legendre's tables, for $\gamma_{1}=73^{\circ} \cdot 9027, F_{1}=2.7024553$

$$
\begin{aligned}
E_{1} & =\overline{\mathrm{r} .0852 \mathrm{I} 67} \\
F_{1}-E_{1} & =\mathrm{I.6172386} \\
\frac{8 \sqrt{A a}}{\sqrt{k_{1}}}=\frac{200}{\sqrt{.960792}} \therefore \frac{8 \sqrt{A a}}{k_{1}}\left(F_{1}-E_{1}\right) & =\frac{M}{\pi}=329.9814 \mathrm{~cm} .
\end{aligned}
$$

Formula 3 and t.-Deries for F and $E$.

Suppose that, in the last example, we calculate $F$ and $E$ by means of formulæ 3 and 4 , instead of taking them from Legendre's tables.

$$
\text { Example 3: } A=a=25, d=\mathrm{I} \text {. }
$$

First for $F$ :

$$
k^{\prime 2}=\mathrm{I}-k^{2}=\mathrm{I}-\frac{25 \mathrm{OO}}{25 \mathrm{OI}}=\frac{\mathrm{I}}{250 \mathrm{I}}
$$

$$
\begin{aligned}
\log \frac{4}{k^{\prime}}=\frac{\mathrm{I}}{2} \log _{k^{\prime 2}} \frac{\mathrm{I} 6}{2} \log _{e} 400 \mathrm{I} 6=.000200+\log _{e} 200 & =5.2985 \mathrm{I} 7 \\
\frac{\mathrm{I}}{4} k^{\prime 2}\left(\log _{k^{\prime}}^{4}-\mathrm{I}\right) & =\underline{.000430} \\
F & =5.298947
\end{aligned}
$$

Second for $E$ :

$$
\begin{aligned}
\mathrm{I}+\frac{k^{\prime 2}}{2}\left(\log \frac{4}{k^{\prime}}-\frac{\mathrm{I}}{2}\right) & =\mathrm{I} .000960 \\
\frac{3 k^{\prime 4}}{\mathrm{I} 6}\left(\log \frac{4}{k^{\prime}}-\frac{\mathrm{I} 3}{\mathrm{I} 2}\right) & =.000000 \\
\therefore E & =\mathrm{I.000960}
\end{aligned}
$$

If these values of $F$ and $E$ be substituted in formula (I), $k$ being 0.9998002 , we obtain the same value of $M$ as by formula (2).

Example 4: $A=25, a=2 \mathrm{O}, d=\mathrm{ro} \mathrm{cm} . \quad$ (See Fig. I.)

$$
k^{2}=\frac{4 \times 20 \times 25}{(45)^{2}+(\mathrm{IO})^{2}}=\frac{\mathrm{I} 6}{\mathrm{I} 7} \therefore k^{\prime 2}=\frac{\mathrm{I}}{\mathrm{I} 7}
$$

$\log \frac{4}{k^{\prime}}=\frac{I}{2} \log (I 6 \times I 7)=\frac{I}{2} \log _{e} 272$

$$
=2.8029010
$$

$$
\begin{array}{ll}
\frac{k^{\prime 2}}{4}\left(\log \frac{4}{k^{\prime}}-\mathrm{r}\right) & =.0265 \mathrm{I} 32 \\
\frac{9 k^{\prime 4}}{64}\left(\log \frac{4}{k^{\prime}}-\frac{7}{6}\right) & =.0007962
\end{array}
$$




$$
\begin{aligned}
& \frac{25 k^{\prime 6}}{256}\left(\log \frac{4}{k^{\prime}}-\frac{\text { III }}{90}\right)=.00003 I 2 \\
& \frac{1225 k^{\prime 8}}{16384}\left(\log \frac{4}{k^{\prime}}-\mathrm{I.27}\right)=. \text {.00000 I4 } \\
& \therefore F=2.8302430 \\
& \mathrm{I}+\frac{k^{\prime 2}}{2}\left(\log \frac{4}{k^{\prime}}-\frac{\mathrm{I}}{2}\right)=\mathrm{I} .0677324 \\
& \frac{3 k^{\prime 4}}{\mathrm{I} 6}\left(\log \frac{4}{k^{\prime}}-\frac{\mathrm{I} 3}{\mathrm{I} 2}\right)=.00 \operatorname{II} 5^{6} \\
& \frac{\mathrm{I} 5 k^{\prime 6}}{\mathrm{I} 28}\left(\log \frac{4}{k^{\prime}}-\mathrm{I} .20\right)=.000038 \mathrm{I} \\
& \frac{\mathrm{I} 75^{k^{\prime 8}}}{2048}\left(\log \frac{4}{k^{\prime}}-\mathrm{I.25}\right)=.00000 \mathrm{I} 7 \\
& \therefore E=\mathrm{I} .0688878
\end{aligned}
$$

To find the value of $M$ we now use equation (I).

$$
\begin{aligned}
k=\sqrt{\frac{16}{\mathrm{I} 7}} & =0.9701425 \\
\frac{2}{k}-k & =1.0914105 \\
& \left(\frac{2}{k}-k\right) F \\
\frac{2}{k} \cdot E & =3.088957 \\
\text { Difference }=\left\{\left(\frac{2}{k}-k\right) F-\frac{2}{k} E\right\} & =0.885385 \\
\text { Multiplying by } 4 \sqrt{A a}=\frac{4 \sqrt{500}}{M} & \text { gives } \\
\frac{\pi}{\pi} & =79.19 \mathrm{I} 50 \mathrm{~cm} .
\end{aligned}
$$

Formula 5.-Weinstein. For any coaxial circles; series more convergent for circles not far apart.

Example 5: $A=30, \quad a=25, \quad d=0$.

Two coplanar circles.

$$
k^{2}=\frac{4 \times 3 O \times 25}{(3 O+25)^{2}}=\frac{I 2 O}{I 2 I}
$$




$$
\begin{aligned}
& k^{\prime 2}=\frac{\mathrm{I}}{\mathrm{I} 2 \mathrm{I}} \quad k^{\prime}=\frac{\mathrm{I}}{\mathrm{II}}, \frac{4}{k^{\prime}}=44 \\
& \log _{e} 44-\mathrm{I}=2.784 \mathrm{I} 90 \\
& \mathrm{I}+\frac{3 k^{\prime 2}}{4}+\frac{33 k^{\prime 4}}{64}+\frac{107 k^{\prime 6}}{256}=\mathrm{I.0052} 34 \\
& \text { Product }=2.801546 \\
& \mathrm{I}+\frac{\mathrm{I} 5 k^{\prime 4}}{\mathrm{I} 28}+\frac{\mathrm{I} 85 k^{k^{6}}}{\mathrm{I} 53^{6}}=\underline{\mathrm{I}} .000008 \\
& \text { Difference }=\text { r.80I } 53^{8} \\
& 4 \pi \sqrt{A \alpha}=4 \pi a \sqrt{\text { I. } 2} \\
& \therefore \frac{M}{\pi}=\mathrm{I} 97 \cdot 3485 \mathrm{~cm}
\end{aligned}
$$

Example 6: Take the same circles as in example 4.

$$
\begin{array}{rlrl}
A & =25, \quad a=2 \mathrm{O}, \quad c=5, \quad d=\mathrm{IO} . \\
k^{\prime 2} & =\frac{\mathrm{I}}{\mathrm{I} 7}, \log \frac{4}{k}-\mathrm{I}=\mathrm{I} .80290 \mathrm{I} \\
\mathrm{I}+\frac{3}{4} k^{\prime 2} & =\mathrm{I} .044 \mathrm{II} 76 \quad \mathrm{I}+\frac{\mathrm{I} 5}{\mathrm{I} 28} k^{\prime 4}=\mathrm{I.0004053} \\
\frac{33}{64} k^{\prime 4} & =.00 \mathrm{I} 7842 & \frac{\mathrm{I} 85}{\mathrm{I} 536} k^{\prime 6}= & .0000245 \\
\frac{\mathrm{IO} 7}{256} k^{\prime 6} & =.000085 \mathrm{I} & \frac{7465}{65536} k^{\prime 8}=\frac{.00000 \mathrm{I} 2}{\mathrm{I} .00043 \mathrm{IO}}=C \\
\frac{59 \mathrm{I} 3}{\mathrm{I} 63^{84}} k^{\prime 8} & =.0000042 \\
\mathrm{Sum} & =\mathrm{I} .04599 \mathrm{II}=B \\
B \log \left(\frac{4}{k^{\prime}}-\mathrm{I}\right) & =\mathrm{I} .8858 \mathrm{I} 84
\end{array}
$$$$
\left\{B \log \left(\frac{4}{k^{\prime}}-\mathrm{I}\right)-C\right\}=0.8853874
$$

Multiplying by $4 \sqrt{500}$ gives $\frac{M}{\pi}=79$. I9I $5 \mathrm{~cm}$, agreeing with value found by other method. 
Formula 6.- Nagaoka. Using q-series, for circles not near each other.

Example 7: $A=a=25, \quad d=20$

$$
\begin{aligned}
\sqrt{k^{\prime}} & =\sqrt{\cos \gamma}=\left(\frac{20}{\sqrt{2900}}\right)^{\frac{1}{2}}=0.6094 \mathrm{I} 83 \\
\frac{l}{2} & =\frac{\mathrm{I}-\sqrt{k^{\prime}}}{2\left(\mathrm{I}+\sqrt{\left.k^{\prime}\right)}\right.}=\frac{\mathrm{I}}{2} \frac{0.39058 \mathrm{I} 7}{\mathrm{I} .6094 \mathrm{I} 83}=0 . \mathrm{I} 2 \mathrm{I} 3425
\end{aligned}
$$

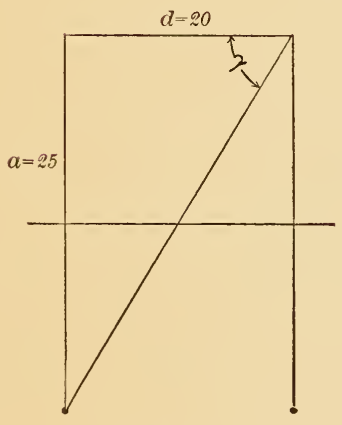

Fig. 11

$\log (I+\epsilon)=0.0002773$

$$
\log q^{\frac{3}{2}}=\overline{2} .6263018
$$

$\log 16 \pi \sqrt{A a}=3.0992099$

$$
\log \frac{M}{\pi}=\mathrm{I} .7257890 \therefore \frac{M}{\pi}=53.1850 \mathrm{cms} \text {, as found by for- }
$$
mula I, Example I.

Formula 7.-Nagaoka. Using q-series, for coaxial circles near each other.

Example 8: $\quad A=a=25, \quad d=4$

$$
\begin{gathered}
k=\sin \gamma=\frac{50}{\sqrt{25 \mathrm{I} 6}} \sqrt{k}=.99840637 \\
l_{1}=\frac{\mathrm{I}-\sqrt{k}}{\mathrm{I}+\sqrt{ } k}=\frac{.00 \mathrm{II} 59363}{\mathrm{I} .9984064} ; \frac{l_{1}}{2}=.00039872=q_{1},
\end{gathered}
$$


as $\left(\frac{l_{1}}{2}\right)^{5}$ and higher powers can be neglected.

$$
\begin{aligned}
\frac{\mathrm{I}}{q_{1}}=2508.04, \log _{e}\left(\frac{\mathrm{I}}{q_{1}}\right) & =7.827238 \\
\left(\mathrm{I}-q_{1}+4 q_{1}^{2}\right) & =0.9996019 \\
8 q_{1} & =0.003 \mathrm{I} 8976 \\
\left\{\log _{e}\left(\frac{\mathrm{I}}{q_{1}}\right)\left[\mathrm{I}+8 q_{1}\left(\mathrm{I}-q_{1}+4 q_{1}^{2}\right)\right]-4\right\} & =3.852195=\mathrm{A} \\
\frac{\mathrm{I}}{2\left(\mathrm{I}-2 q_{1}\right)^{2}} & =0.5007985=\mathrm{B} \\
4 \sqrt{A a} & =\underline{\mathrm{IOO}}=\mathrm{C}
\end{aligned}
$$

Product $\mathrm{A} \times \mathrm{B} \times \mathrm{C},=M \pi,=192.9174 \mathrm{~cm}$.

There is a difficulty in using the above formula owing to the fact that when $k$ is nearly unity the numerator of the expression for $l_{1}$ is small, and unless the value of $k$ is carried out to about eight decimal places the value of $M$ may be appreciably in error. For approximate calculations a seven-place table of logarithms is sufficient, and it is not very troublesome to carry out this one number to the necessary number of places for precision calculations. Or, $k$ can easily be computed to any degree of accuracy without logarithms. The same thing applies to formulæ 3 and 4 , where $k^{\prime}$ must be computed with great precision when it is quite small.

Formula 8.-Maxwell. Two circles of unequal radii. Formula accurate only' for circles very near each other.

Example 9: $A=26, \quad a=25, \quad d=\mathrm{I}, \quad c=\mathrm{I}$ and $r=\sqrt{2}$

$$
\begin{gathered}
\text { Since } r=\sqrt{2}, \log _{e} \frac{8 a}{r}=\log \frac{200}{\sqrt{2}}=4.951744 \\
\mathrm{I}+\frac{c}{2 a}=1.020000 \quad 2+\frac{c}{2 a}=2.020000 \\
\frac{c^{2}+3 d^{2}}{\mathrm{I} 6 a^{2}}=.000400 \quad-\frac{3 c^{2}-d^{2}}{\mathrm{I} 6 a^{2}}=-.000200 \\
-\frac{c^{3}+3 c d^{2}}{32 a^{3}}=\frac{-.000008}{\mathrm{I.020392}}=B \quad-\frac{c^{3}-6 c d^{2}}{48 a^{3}}=\frac{-.000010}{2.019790=C}
\end{gathered}
$$




$$
\begin{aligned}
B \log \frac{8 a}{r} & =5.052310 \\
C & =\frac{2.019790}{3.032520} \quad \text { Multiply by } 4 a=\text { IOO and } \\
\left\{B \log \frac{8 a}{r}-C\right\} & =3.032 .252 \mathrm{~cm} .
\end{aligned}
$$

This formula would be less accurate for the circles of problem 4, but is accurate for circles close together, as this problem shows.

Formula 9.-Maxwell. Circles of equal radii near each other.

Example Io: $A=\dot{\alpha}=25, d=\mathrm{I}$

$$
\begin{aligned}
& \frac{8 a}{d}=200 \quad \log _{e} 200=5.2983 \mathrm{I} 7 \\
& \log \frac{8 a}{d} \cdot\left(\mathrm{I}+\frac{3 d^{2}}{\mathrm{I} 6 a^{2}}\right)=\mathrm{I} .000300 \times 5.2983 \mathrm{I} 7=5.29990 \\
&\left(2+\frac{d^{2}}{\mathrm{I} 6 a^{2}}\right)=\frac{2.000 \mathrm{ro}}{3.29980} \\
& \text { Multiply by } 4 a=\mathrm{IO0} \\
& \frac{M}{\pi}=329.980,
\end{aligned}
$$

nearly agreeing with the more exact value found under problem 2 .

This is a very simple and convenient formula for equal circles, and gives approximate results for circles still farther apart than in this problem.

Formula IO.-Coffin. Circles of equal radii, not farther apart than $d=a$.

Example II: $A=a=25, d=\mathrm{I} 6$

$$
\frac{8 a}{d}=\mathrm{I} 2.5 \quad \log _{e} \mathrm{I} 2.5=2.5257286
$$

First series of terms $=B=1.074478$

Second series of terms $=C=2.023220$

$$
\begin{aligned}
& \therefore\left\{B \log \frac{8 a}{d}-C\right\}=0.690620 \\
& 4 a=100 \\
& \therefore \frac{M}{\pi}=69.0620
\end{aligned}
$$


This agrees. with the value given by formula I within one part in 200,000 . As the distance apart of the circles increases the accuracy by this formula of course gradually decreases.

Formula II._Extension of Maxwell's series formula for unequal circles.

Example I2: $A=25, \quad a=20, c=5, \quad d=10$

$$
\begin{aligned}
& r=\sqrt{ } c^{2}+d^{2}=5 \sqrt{5}, \quad \log \frac{8 a}{r}=\log \frac{32}{\sqrt{5}}=\quad 2.6610169 \\
& -\left(2+\frac{c}{2 \alpha}\right)=-2.125000 \\
& -\frac{d^{2}-3 c^{2}}{16 a^{2}}=-.003906 \\
& +\frac{93 d^{1}-534 c^{2} d^{2}-19 c^{4}}{6144 d^{4}}=-.000424 \\
& -\frac{1845 c d^{4}-3030 c^{3} d^{2}-379 c^{5}}{61440 a^{5}}=-.000271 \\
& \begin{aligned}
+\frac{6 c d^{3}-c^{3}}{48} a^{3} & =+.007487 \\
C & =-2.122 \mathrm{II} 4
\end{aligned} \\
& \mathrm{I}+\frac{c}{2 a}=+1 . \mathrm{I} 25000 \\
& \frac{c_{2}+3 d^{2}}{\mathrm{I} 6 a^{2}}=+0.05078 \mathrm{I} \\
& \frac{45 c d^{4}-30 c^{3} d^{2}-19 c^{5}}{2048 a^{5}}=+0.000277 \\
& \frac{3 c d^{2}+c^{3}}{32 a^{3}}=-0.006348 \\
& \frac{\mathrm{I} 5 d^{4}-42 c^{2} d^{2}-\mathrm{I} 7 c^{4}}{1024 a^{4}}=-0.000210 \\
& B=\mathrm{I} . \mathrm{I} 69500 \\
& B \log _{e} \frac{\delta a}{r}=3 \text {. I I } 2060 \\
& C=\frac{\mathrm{I} . \mathrm{I} 22 \mathrm{II} 4}{0.989946}
\end{aligned}
$$

multiplying by $4 \alpha=80, \frac{M}{\pi}=79.1957 \mathrm{~cm}$ 
This result is correct to one part in I9,000 (see examples 4 and 6). Using only the first three terms for $B$ and $C$, the result would be too large by one part in I 750 .

Formula I2.-Extension of Maxuell's series for coplanar circles.

Example I3: $A=25 \quad a=20 \quad c=5 \quad d=0$

$$
\begin{aligned}
& \mathrm{I}+\frac{c}{2 \alpha}=\text { I.I } 25000 \quad-\frac{c^{3}}{32 \alpha^{3}}=-0.000488 \\
& \frac{c^{2}}{16 a^{2}}=0.003906 \quad-\frac{19 c^{5}}{2048 a^{5}}=-\underline{0.000009} \\
& \frac{\mathrm{I} 7 c^{4}}{\mathrm{I0} 24 a^{4}}=\underline{0.000065} \quad B^{\prime}=-0.000497 \\
& \text { + I.I } 2897 \mathrm{I} \quad-\left(2+\frac{c}{2 a}\right)=-2 . \mathrm{I} 25000
\end{aligned}
$$

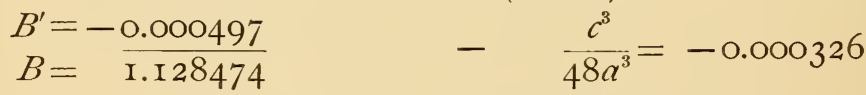

$$
\begin{aligned}
& \log \frac{8 a}{c}=3.4657359 \quad-\frac{\mathrm{Ig}^{4}}{6 \mathrm{I}_{44 a^{4}}}=-\underline{0.0000 \mathrm{I} 2} \\
& B \log \frac{8 a}{c}=\overline{3.910994}-\overline{2.12533^{8}} \\
& C=-2 . \operatorname{II} 36 \text { I } 3 \quad \frac{3 c^{2}}{16 a^{2}}=+ \text { o.oII7I } 9 \\
& \text { Multiply } 4 a=\frac{\mathrm{I} .7973^{8 \mathrm{I}}}{8 \mathrm{o}} \quad \frac{379 c^{5}}{\mathrm{M}}=\frac{\mathrm{I} 43.79048}{6 \mathrm{I} 44 \mathrm{O} a^{5}}=\quad C=\frac{+0.000006}{2 . \mathrm{II} 36 \mathrm{I} 3}
\end{aligned}
$$

\section{MUTUAL INDUCTANCE OF COILS OF RECTANGULAR SECTIONS.}

Formulce 13, 14, I5.-Rowland. Coils of equal radii not very near each other.

Example I4: $A=a=25, b=c=2 \mathrm{~cm}, \quad d=10$.

The mutual inductance of the two coils is $M=M_{0}+\Delta M$.

We find $M_{0}$ by formula I, 6, or Io, and $\Delta M$ by I4 and I 5 .

$$
\begin{aligned}
& M_{0}= \mathrm{I0} 7.4885 \pi \\
& k=\sin \gamma=\frac{50}{\sqrt{2600}}=0.9805807 \\
& k^{2}=.9615383 \\
& \log _{10} F=0.482 \mathrm{I} 754 \\
& \log _{10} E=0.0207625
\end{aligned}
$$


Substituting these values in formula (15) we obtain

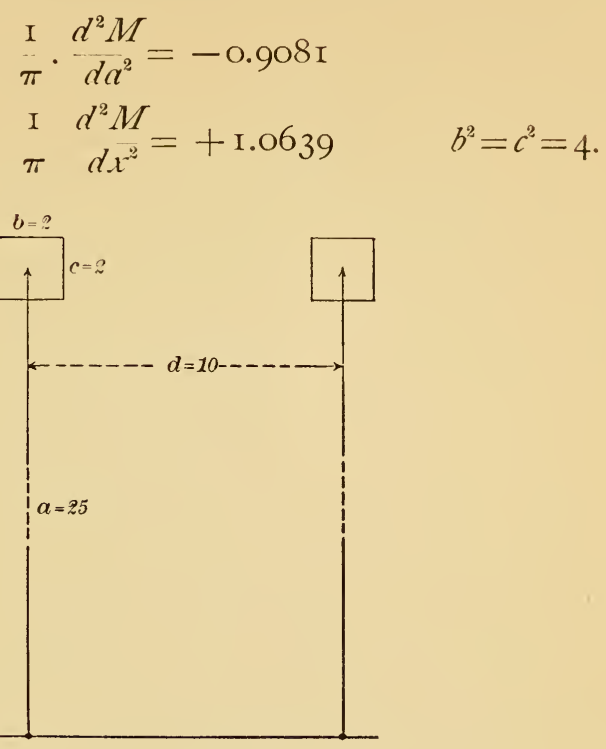

Fig. 12

Substituting these values in formula (14) we obtain

$$
\begin{aligned}
& \Delta M=.05 \mathrm{I} 93 \pi \\
& \therefore M= M_{0}+\Delta M=(\mathrm{IO} 7.4885+.05 \mathrm{Ig}) \pi \\
&=\mathrm{IO} 7.5404 \pi \mathrm{cm} .
\end{aligned}
$$

The correction $\triangle M$ thus amounts to about one part in 2000 of $M$. At a distance $d=20 \mathrm{~cm}$; the correction is over one part in Iooo. For a coil of section $4 \times 4 \mathrm{~cm}$ at $d=\mathrm{IO}, \Delta M$ would be four times as large as the value above, or about I part in 500, and at $20 \mathrm{~cm}$ I part in 250.

Formulce 18 and 19.-Rayleigh. Coils of equal or unequal radii, not very near each other.

$$
\text { Example 1 } 4 a: A=a=25, b=4, c=\mathrm{I}, d=\mathrm{IO}
$$

We now find by formula (I) in accordance with formula (I9) the mutual inductance of the following pairs of circles: O, I when $a=$ 25, $A=25 \cdot 5, d=$ ro.

$$
16360-07-6
$$


$\mathrm{O}, 4$ when $a=25, A=24.5, d=\mathrm{IO} ; \mathrm{O}, 2$ when $a=A=25$ and $d=$ 8; $\mathrm{O}, 3$ when $A=a=25, d=\mathrm{I} 2$, and finally $\mathrm{O}, \mathrm{O}^{\prime}$ when $A=a=25$, $d=\mathrm{IO}$, Thus:

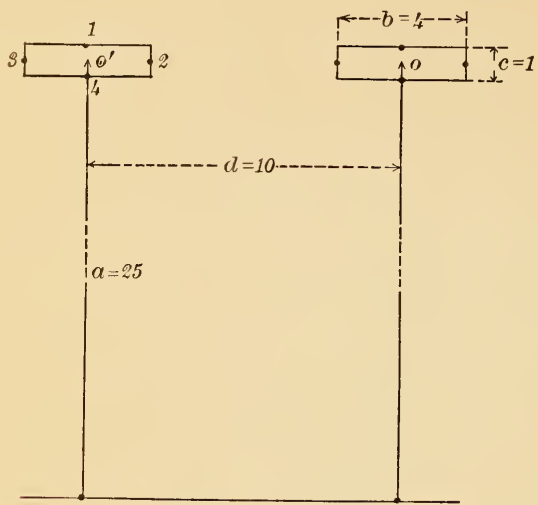

Fig. 13

$$
\begin{aligned}
M_{1} & =109.3217 \pi \\
M_{4} & =105.4287 \pi \\
M_{2} & =\mathrm{I} 27.3949 \pi \\
M_{3} & =\frac{91.9206 \pi}{434.0659 \pi} \\
M_{0} & =\frac{107.4885 \pi}{326.5774 \pi} \\
\therefore M & =108.859 \mathrm{I} \pi \\
M_{0} & =107.4885 \pi \\
\frac{\Delta M}{\pi}= & \mathrm{I} .3706 \mathrm{~cm} .
\end{aligned}
$$

Formula 22 and 23.-Lyle. For coils of square section of equal or unequal radii, not very near one another; also for coils of rectangular section not square.

The equiwalent radius $r=a\left(\mathrm{I}+\frac{b^{2}}{24 a^{2}}\right)$

Example I 5: $A=a=25 \mathrm{~cm}, \quad b=c=2 \mathrm{~cm}, d=10 \mathrm{~cm}$.

$$
r=25\left(\mathrm{I}+\frac{4}{\mathrm{I} 5000}\right)=25.00667 \mathrm{~cm} \text {. }
$$

$M$ is now found by using formula $\mathrm{I}, 6$, or Io, employing $r$ in place of $a$ as the radius.

The result is $M=107.5402$, agreeing very closely with the result found under example 14 .

$$
M-M_{0}=\Delta M=.05 \mathrm{I} 7 .
$$

Example 16: $A=a=25, b=4, c=\mathrm{I}, \quad d=\mathrm{IO}$

$$
r=25\left(\mathrm{I}+\frac{\mathrm{I}}{\mathrm{I} 5000}\right)=25.00167
$$

$\beta^{2}=\frac{b^{2}-c^{2}}{\mathrm{I} 2}=\frac{\mathrm{I} 5}{\mathrm{I} 2}=\mathrm{I} .25,2 \beta=2.236 \mathrm{~cm}$, the distance apart of the 
two filaments which replace the coil. We now find by formula I, 6 , or ro the mutual inductances of two circles $I, 2$ on the two circles 3,4 , where $a=25.00167$ and $d$ is 7.764 , ro and $12.236 \mathrm{~cm}$, respectively. Thus:

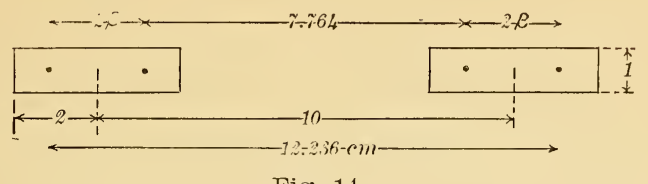

Fig. 14

$$
\begin{aligned}
2 M_{13} & =2 \mathrm{I} 5.00228 \pi \\
M_{14} & =90.3 \mathrm{I} 304 \pi \\
M_{23} & =\mathrm{I} 3 \mathrm{O} .14060 \pi \\
4 M & =435.45592 \pi \\
\therefore M & =\mathrm{I0} 8.8640 \pi \\
M_{0} & =107.4885 \pi \\
\Delta M & =\mathrm{I} .3755
\end{aligned}
$$

$\Delta M=$ the correction for section of the coils whose dimensions are given above. These values of $M$ and $\Delta M$ agree nearly with the results obtained in Example I $4 a$ above.

Formula 24.-For LM. Radii of coils equal, square section.

Eixample I7: $A=a=25, \quad d=$ Io,$\quad b=c=2$

$$
\begin{gathered}
\log \frac{8 a}{d}-\mathrm{I}=\mathrm{I} .9957 \\
-\frac{3 d^{2}}{\mathrm{I} 6 a^{2}}\left(\log \frac{8 a}{d}-\frac{4}{3}\right)=-\frac{0.0499}{\mathrm{I} .945^{8}} \\
\frac{b^{2}}{6 a}=\frac{4}{\mathrm{I} 5 \mathrm{O}} \\
\therefore \frac{\Delta M}{\pi}=.05 \mathrm{I} 4 \text { agreeing closely with the }
\end{gathered}
$$

result in Example 15.

Formula 2,a.-For $\Delta M$. Radii of the coils unequal, section square.

Example 18: $A=25, a=20, d=6, b=2$ (section $2 \times 2 \mathrm{~cm}$ ) Substituting in formula $24 a$ we obtain

$$
\Delta M=.0740
$$


Using Lyle's method of computing $M$ we obtain

$$
\begin{aligned}
M & =106.930 \\
M_{0} & =\mathrm{I06.855} \\
\Delta M & =.075
\end{aligned}
$$

This difference amounts to one part in Ioo, ooo of the value of $M$. Formula (24a) is less convenient than (22) when one wants only the value of $M$. It is, however, valuable as a check when the coils are not far apart. It is not reliable for coils at a distance.

Formula 25.-Stefan. For coils of equal radii, and rectangular section.

This formula has been shown to be in error, but is substantially right for coils near together. We give in Fig. I 5 the results of calculations for coils $2 \times 2 \mathrm{~cm}$ section at different distances, showing how much formula 25 is in error. No numerical example will be given here as this formula should not be employed. Figure i 5 shows the magnitude of the error in this formula for one particular pair of coils.

Formula 26.-Rosa. Coils of equal radii. Most accurate of all the formula for coils near together (but not in contact), and less accurate when coils are far apart.

Example 19: $A=a=25, \quad b=4, c=\mathrm{I}, \quad d=\mathrm{IO}$

(same coils as examples $14 \alpha, \mathrm{r} 6$ ).

$$
\begin{gathered}
\log _{d} \frac{8 a}{d}=\log _{e} 20=2.9957 \\
\frac{3 b^{2}+c^{2}}{96 a^{2}} \cdot \log \frac{8 a}{d}=\frac{49 \times 2.9957}{60,000}=.0024465 \\
\frac{b^{2}-c^{2}}{\mathrm{I} 2 d^{2}}=\frac{\mathrm{I} 5}{\mathrm{I} 200}=.0125000 \\
\frac{2 b^{4}+2 c^{4}-5 b^{2} c^{2}}{\mathrm{I} 2 \mathrm{O} d^{4}}=\frac{434}{\mathrm{I}, 200,000}=.00036 \mathrm{I} 7
\end{gathered}
$$




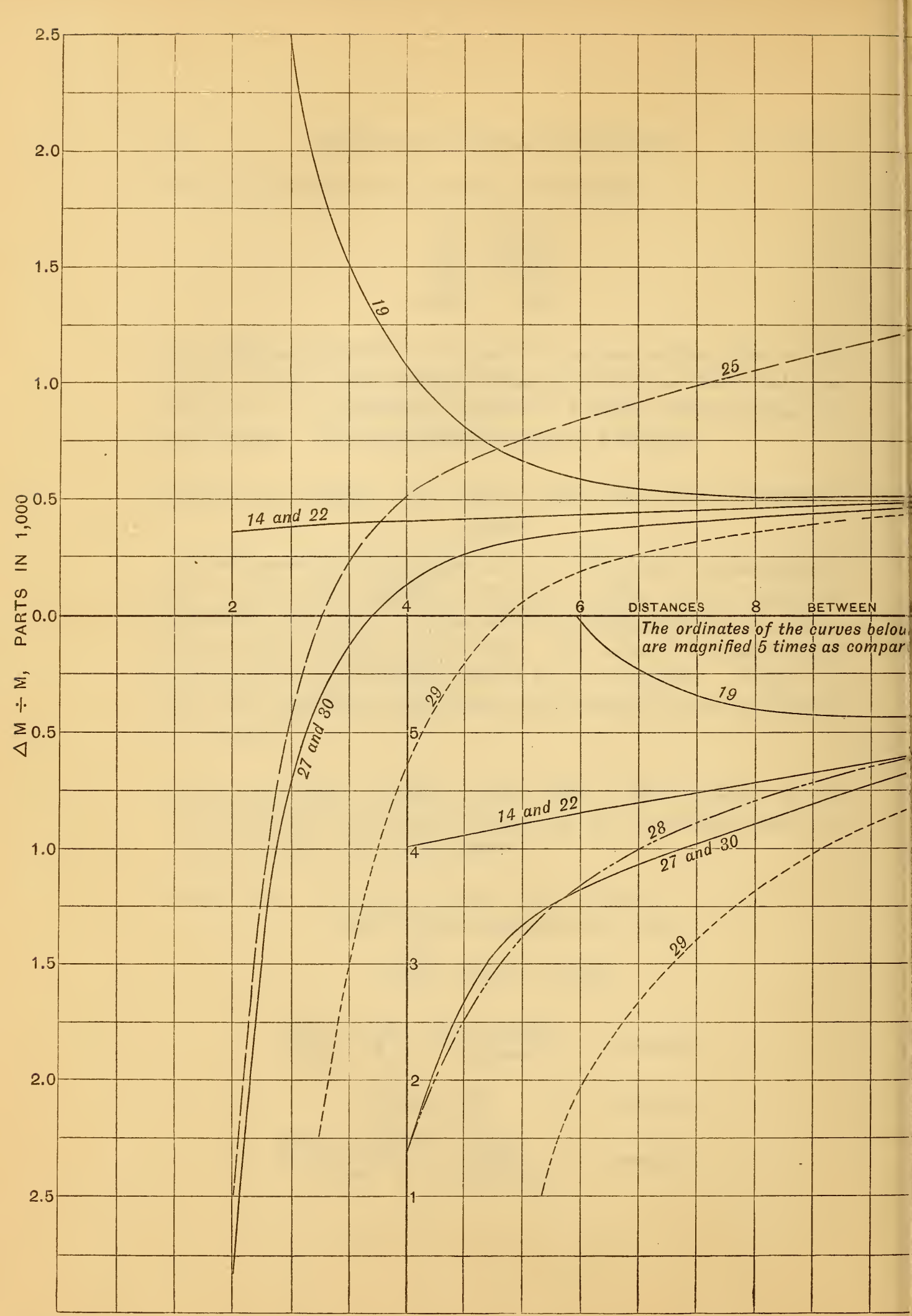

Fig. 15.-Comparison of Formulae for a pair of coils of equal radii $(25 \mathrm{~cm})$ and equal section $(2 \times 2 \mathrm{~cm})$ at varying distances I6360-07. (To follow page 40I.) 


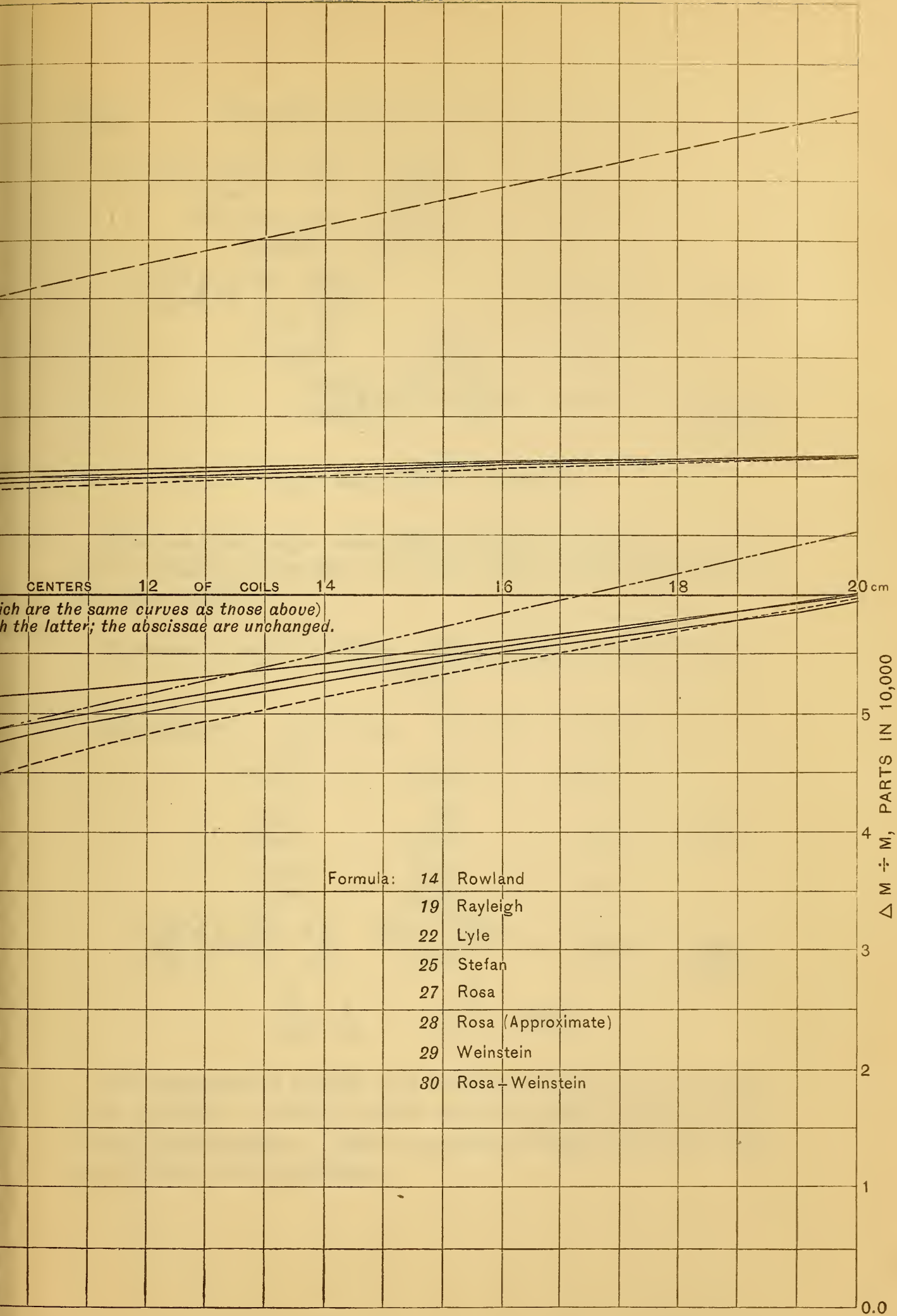

, $20 \mathrm{~cm}$, center to center). Corrections $\frac{\Delta M}{M}$ are negative below the axis. The curve representing Formulae 27 and 30 e part in 100,000 of $M$. 


$$
\begin{aligned}
& \frac{3 b^{6}-3 c^{6}+14 b^{2} c^{4}-14 b^{4} c^{2}}{504 d^{6}}=\frac{8925}{504 \times 10^{6}}=.0000177 \\
& \frac{6 b^{4}+6 c^{4}+5 b^{2} c^{2}}{5760 a^{2} d^{2}}=\frac{1622}{360 \times 10^{6}}=.0000045 \\
& \frac{7 c^{2} d^{2}}{\mathrm{IO} 24 a^{4}}\left(\log \frac{8 a}{d}-\frac{\mathrm{I} 63}{84}\right)=\quad=.0000 \mathrm{I} 8=.0153322 \\
& -\frac{\text { I I } b^{2}-3 c^{2}}{\text { I } 92 a^{2}}=-\frac{\text { I } 73}{\text { I } 20,000}=-.00144 \mathrm{I} 7 \\
& -\frac{{ }_{1} 5 b^{2} d^{2}}{{ }_{\text {IO } 24 a^{4}}}\left(\log \frac{8 a}{d}-\frac{97}{60}\right)=-.0000827=-\frac{.00 I 5244}{.0158078} \\
& 4 a=\mathrm{I} 00 . \frac{\Delta M}{\pi}=\mathrm{I} \cdot 3808 \mathrm{~cm}
\end{aligned}
$$

This is a little larger value than found by formula I9 and 23, and we shall see later that it is more nearly correct than either of the other values.

Formulce 27 and 28.-Rosa. Coils of equal radii and square section.

Example 20: $\quad A=a=25, \quad b=c=2, \quad \mathrm{~d}=\mathrm{IO}$

$$
\begin{gathered}
\log \frac{8 a}{d}-\mathrm{I}=2.9957-\mathrm{I}=\mathrm{I.9957} \\
\frac{\mathrm{I} 7 b^{2}}{240 d^{2}}=\frac{68}{24,000}=.0028=\mathrm{I.9985} \\
\frac{-a^{2} b^{2}}{5 d^{4}}=-\frac{2500}{50,000}=-.0500 \\
\frac{-3 d^{2}}{\mathrm{I} 6 a^{2}}\left(\log \frac{8 a}{d}-\frac{4}{3}\right)=\frac{300 \times \mathrm{I.6624}}{\mathrm{I0,000}}=-.0499=-\frac{.0999}{\mathrm{I.8986}} \\
\frac{b^{2}}{6 a}=\frac{4}{\mathrm{I} 50} \quad \therefore \frac{\Delta M}{\pi}=.05063
\end{gathered}
$$

The approximate formula (28) would have given .0519 (agreeing with forinulæ I4 and 22), which would be amply accurate for any experimental purpose. When the section is larger these sniall terms are, however, more important. 
Example 21: $A=a=25, \quad b=c=5, \quad d=\mathrm{IO}$

$$
\begin{aligned}
& \log \frac{8 a}{d}-\mathrm{I} \quad=\mathrm{I} .9957 \\
& \frac{17 b^{2}}{240 d^{2}} \quad=.0177=2.0134 \\
& \frac{-a^{2} b^{2}}{5 d^{4}} \quad=-.3 \text { } 25 \\
& -\frac{3 d^{2}}{16 a^{2}}\left(\log \frac{8 a}{d}-\frac{4}{3}\right)=-\underline{.0499}=-.3624 \\
& \frac{b^{2}}{6 a}=\frac{25}{\mathrm{I} 5 \mathrm{O}} \\
& \therefore \frac{\Delta M}{\pi}=0.275^{2} \\
& M_{0}=\mathrm{I07.4885} \text { (see example I4.) } \\
& M=\overline{\mathrm{I07.7637}} \mathrm{cm} \text {. }
\end{aligned}
$$

This is a very simple formula for computing $\Delta M$, and within a considerable range (i. e., $d$ not larger than $a$ and yet the coils not in contact) it is very accurate.

Formula 29.-Weinstein. Formula for mutual inductance of equal coils, in elliptic integrals.

This formula is very accurate for coils not near together, but is much less accurate for coils relatively near. See Fig. I5. The revised form of this formula ( 30$)$ is more accurate as well as more convenient. and hence no example will be given of (29).

Formula 30.-Rosa-Weinstein. For $\Delta M$, for two coils of equal radii and equal section.

Example 22: $A=a=25, \quad b=4, \quad c=\mathrm{I}, \quad d=\mathrm{IO}$

$$
\begin{aligned}
a_{1} & =\mathrm{I} 5.0000533 & \sin ^{2} \gamma & =\frac{2500}{2600}=\frac{25}{26} \\
a_{2} & =0.0020267 & \cos ^{2} \gamma & =\frac{100}{2600}=\frac{\mathrm{I}}{26} \\
a_{3} & =0.2 \mathrm{I} 7 & \frac{c^{2}}{24 a^{2}} & =.0000667
\end{aligned}
$$




$$
\begin{aligned}
a_{1}-a_{2}-a_{3}+\left(2 a_{2}-3 a_{3}\right) \cos ^{2} \gamma+8 a_{3} \cos ^{4} \gamma & =\mathrm{I} 4.7587 \mathrm{I} 20 \\
a_{1}+\frac{a_{2}}{2}+2 a_{3}+\left(2 a_{2}+3 a_{3}\right) \cos ^{2} \gamma+8 a_{3} \cos ^{4} \gamma & =\mathrm{r} 5.4628292 \\
A=0.0004730 \quad \text { Also } F & =3.035 \mathrm{II} 68 \\
E & =\mathrm{I} .0489686 \\
B=0.012390 \mathrm{I} & =0.00107 \mathrm{I} 9 \\
(F-E)\left(A+\frac{c^{2}}{24 a^{2}}\right) & =0.0129968 \\
E B & =0.0140687 \\
\text { Sum } & =1.3795 \mathrm{~cm} .
\end{aligned}
$$

This is not as simple to calculate as (26) and when $d$ is less than $a / 2$ is less accurate than (26). But for $d=a$ or greater it is more accurate than (26), and indeed the most accurate of all the formulæ.

Formula 3I.-Mutual inductance in terms of self-inductance.

This is for coils relatively near. An example will be given below, page 408 .

Formula 32.-Mutual inductance by geometric mean distances.

$$
\text { Example 23: } \begin{aligned}
A & =25 . \mathrm{I} \\
a & =25.0 \\
b & =c=0 . \mathrm{I} \mathrm{cm} \\
d & =0 . \mathrm{Im}
\end{aligned}
$$

The geometrical mean distance of two coils, corner to corner, as in Fig. IO, is 0.9977 Or, and $\log \frac{r}{R}=0.002302$

$$
\begin{aligned}
\therefore \frac{\Delta M}{\pi} & =100 \times 0.002302(\mathrm{I} .002) \\
& =0.2307 .
\end{aligned}
$$

COMPARISON OF SEVEN FORMULE FOR COILS OF SQUARE SECTION.

$$
\text { I4, I9, 22, 25, 27, 29, 30. }
$$

We have obtained above by different formulæ different values of the correction $\Delta M$. In order to show the degree of divergence among them for a particular pair of coils as the distance between 


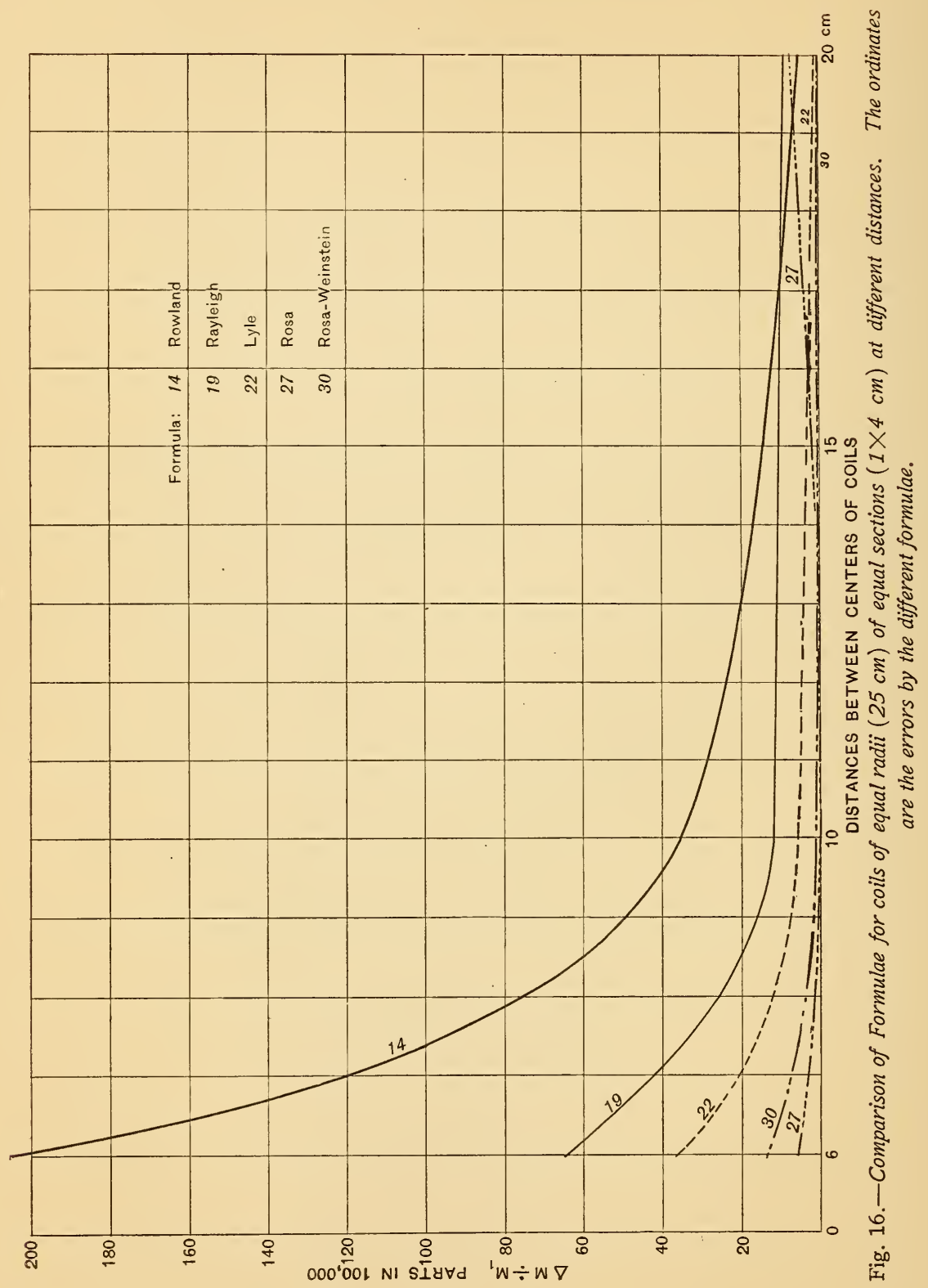


the coils is varied we have made a series of computations by seven different formulæ for two coils of square section, $2 \times 2 \mathrm{~cm}$, and equal radii $(\alpha=25 \mathrm{~cm})$, for distances varying between 3 and $20 \mathrm{~cm}$. The results are plotted in Figure 15 . Formulæ 27 and 30 give the values of $\frac{\Delta M}{M}$ with great precision and agree so closely that they are represented by the same line almost to $d=20 . \quad \Delta M$ is negative for small distances, and at abont $d=3.6 \mathrm{~cm}$ becomes zero, increasing gradnally as the distance increases to $20 \mathrm{~cm}$. $\Delta M$ decreases after passing a maximum at about $6 \mathrm{~cm}$, but $M$ decreases faster than $\Delta M$, hence the relative value of the correction is greater at $20 \mathrm{~cm}$ than at any point between that and $3 \mathrm{~cm}$. This is true only for coils of square section. As the correction for sixth differentials is zero for a coil of square section, formulæ 27 and 30 agree for small distances, which they do not quite do for coils not of square section. The dotted curves show how formulæ 25 and 29 diverge from the true values given by 27 and 30 . Rowland's and Lyle's formulæ (I4) and (22) agree almost exactly, both being based on the same assumptions. They are very accurate for coils not near each other. Formula (I9) departs farthest from the true values at small distances. The uncertainty of the value of $M=M_{0}+\Delta M$ for these coils as given by formulæ 27 and 30 is not as much as one in a hundred thousand.

COMPARISON OF FIVE FORMULE FOR COILS NOT OF SQUARE SECTION.

$$
\text { I4, I9, 22, 27, } 30 \text {. }
$$

For coils of section not square the corrections are much larger and the formulæ are not so accurate. We have found differences in some of the examples above for the coils of sections $4 \times \mathrm{x}$ c $\mathrm{m}$ and we will now decompose these coils into four coils each of square section, and calculate $\triangle M$ by means of formula (27) which we have seen agrees with (30) and is very accurate. If the two coils A and $\mathrm{B}$ be considered to be made up of four coils each, the mutual inductance of $A$ on $B$ will be the sum of the mutual inductances of the four constituents of $\mathrm{A}$ on the four separate coils of $\mathrm{B}$. Thus, if $M_{\mathrm{AB}}$ be the mutual inductance of $\mathrm{A}$ on $\mathrm{B}$ and $M_{45}$ that of coil 4 on coil 5 , etc., we see that 


$$
\begin{aligned}
M_{\mathrm{AB}} & =M_{45}+2 M_{35}+3 M_{25}+4 M_{15}+3 M_{16}+2 M_{17}+M_{18} \\
\text { or, } M_{\mathrm{AB}} & =M_{7}+2 M_{8}+3 M_{9}+4 M_{10}+3 M_{11}+2 M_{12}+M_{13}
\end{aligned}
$$

where in the second expression $M_{7}$ means the mutual inductance of two coils distant $7 \mathrm{~cm}$ between centers, as coils 4 and 5 are, etc. Calculating $M_{0}$ and $\Delta M$ for each of the seven distances from 7 to I $3 \mathrm{~cm}$ inclusive, and substituting in equation $(x)$ above we shall have $M_{\mathrm{AB}}$ the mutual inductance of the two coils to a very high degree of accuracy. These values are given in the accompanyiug

\begin{tabular}{|c|c|c|}
\hline \multirow{4}{*}{ At } & $M$ & $\Delta M$ \\
\hline & $2 \frac{\pi}{\pi}$ & $\pi$ \\
\hline & $7 \mathrm{~cm} \mathrm{I} 39.6579$ &. $\mathrm{OI} 5^{\mathrm{I}}$ \\
\hline & I $27 \cdot 3947$ & .0144 \\
\hline & I I $6.785^{2}$ & .0136 \\
\hline IC & 107.4885 & .0129 \\
\hline I I & 99.2607 & .0122 \\
\hline I 2 & 91.9204 & .0116 \\
\hline I 3 & $85.329 \mathrm{I}$ & .OIII \\
\hline
\end{tabular}
table :

$$
\begin{aligned}
& \frac{B I}{\pi} \\
& I 39.6730 \times I=I 39.6730 \\
& \text { I } 27.409 \mathrm{I} \times 2=254.8182 \\
& \text { I } 6.7988 \times 3=350.3964 \\
& \text { I07.50I } 4 \times 4=430.0056 \\
& 99.2729 \times 3=297.8187 \\
& 9 \mathrm{I} .932 \mathrm{O} \times 2=\mathrm{I} 83.8640 \\
& 85.3402 \times \mathrm{I}=85.3402 \\
& \Sigma(M \pi)=\mathrm{I} 74 \mathrm{I} .9 \mathrm{I} 6 \mathrm{I} \\
& \Sigma(M \pi) \div \mathrm{I} 6=\mathrm{I0} 8.8698 \\
& M_{0} \pi=107.4885 \\
& \Delta M \div \pi=\mathrm{I} \cdot 38 \mathrm{I} 3 \\
& \text { Calculated above by (27), Example I9, } \Delta M \div \pi=\quad \text { I.3808 } \\
& =.0005
\end{aligned}
$$

This difference of five parts in a million of the value of $M_{A B}$ is an extremely small discrepancy. The value calculated by the method of decomposing the coil into several coils of square section is very accurate if the several values of $M$ are calculated with sufficient accuracy, and we may be sure of the value above to less than one part in a million, as the average value of the corrections $\Delta M$ for the separate square sections is less than a hundredth part of $\triangle M$ for the whole coils $A, B$. The result by formula (30) differs slightly from that by (27), being .oor 3 less.

This difference is mainly due to the sixth differentials, neglected in (30), which example (19) shows to be .oor8. If we add this to the value obtained by (30) we have I.38I 3 , agreeing exactly with 


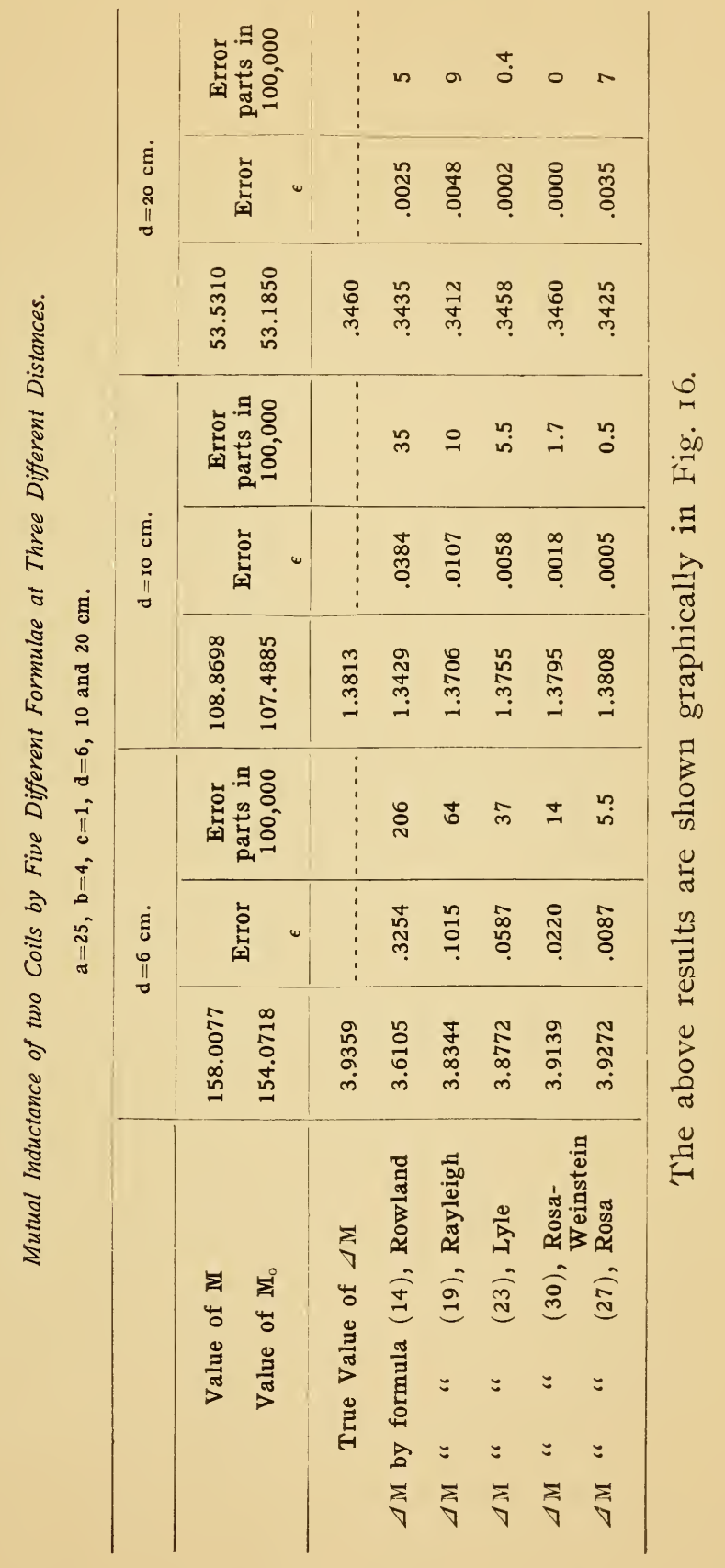


the value above by the more laborious method of decomposition. This close agreement of (27) and (30), two expressions derived by different processes from two entirely different formulae, is very satisfactory.

Lyle's formula (22) for this case gives a value too small by $.005^{8}$, Rayleigh's (I9) too small by .0107, and Rowland's (I4) too small by .0384 . The accompanying table gives these results together with corresponding values for $d=6$ and $d=20$. Formula (27) begins to be in error before $d=20$, but for the smaller distances it is the most accurate of all. Formulæ (27) and (30) supplement each other, and taken together they will give reliable results for all distances except when the coils are nearly in contact. When the coils are in contact or nearly so, formulae (3I) and (32) are to be used.

COILS OF ONE LAYER. CURRENT SHEETS.

Formulæ 26 and 30 apply when the depth is very small compared with the breadth of the coil, as in a single layer coil. In such a case the other formulæ are scarcely applicable, as the error increases rapidly with a great divergence from square section. If we make $c=0$, as in a current sheet we can get an interesting check on the results of the formulæ by using the formula for the self-inductance of current sheets, deriving from the values of several self-inductances the mutual inductance in question. Suppose we have two coaxial current sheets $\mathrm{A}, \mathrm{B}$ each $5 \mathrm{~cm}$ long and with a radius of $25 \mathrm{~cm}$, their centers being Io $\mathrm{cm}$ apart. If a third current sheet $\mathrm{C}$ fill the gap between them it would be $5 \mathrm{~cm}$ long. Let $L_{\mathrm{A}}$ be the self-inductance of $\mathrm{A}$, which is also that of $\mathrm{B}$ or $\mathrm{C}, L_{\mathrm{AB}}$ be the self-inductance of two sections $\mathrm{AC}$ together (or $\mathrm{CB}$ together) counting $\mathrm{AC}$ as two turns in series, and $L_{\mathrm{ABC}}$ be the self inductance of the three together in series. Also let $M_{\mathrm{AC}}$ be the mutual inductance of $\mathrm{A}$ on $\mathrm{C}$ and $M_{\mathrm{AB}}$ be the mutual inductance of $\mathrm{A}$ on $\mathrm{B}$. We wish to find $M_{\mathrm{AB}}$. Remembering that the self-inductance of a coil of several turns is equal to the sum of the several self-inductances plus the mutual inductances of the several turns on one another, we see that:

$$
\begin{aligned}
L_{\mathrm{ABC}} & =3 L_{\mathrm{A}}+4 M_{\mathrm{AC}}+2 M_{\mathrm{AB}} \\
\text { A1so } L_{\mathrm{AB}} & =2 L_{\mathrm{A}}+M_{\mathrm{AC}} \\
\therefore \quad L_{\mathrm{ABC}} & =3 L_{\mathrm{A}}+2\left(L_{\mathrm{AB}}-2 L_{\mathrm{A}}\right)+2 M_{\mathrm{AB}} \\
\text { or, } M_{\mathrm{AB}} & =\frac{L_{\mathrm{ABC}}+L_{\mathrm{A}}-2 L_{\mathrm{AB}}}{2}
\end{aligned}
$$


If we calculate the three self-inductances $L_{\mathrm{ABC}}, L_{\mathrm{AB}}$, and $L_{\mathrm{A}}$, for the current sheets by an accurate formula we can obtain $M_{\mathrm{AB}}$, and thus obtain a check on the values calculated for the same case by (27) and (30).

These self-inductances may be calculated with great precision by the formula of Coffin ${ }^{19}$ or of Lorentz. ${ }^{19}$ The results are as follows, taking the above dimensions, with 50 turns of thin tape I $\mathrm{mm}$ wide on each coil:

$$
\begin{aligned}
L_{\mathrm{ABC}} & =4,774,460 \pi \\
L_{\mathrm{A}} & =798,457 \pi \\
\mathrm{Sum} & =5,572,9 \mathrm{I} 7 \pi \\
2 L_{\mathrm{AB}} & =5,023,808 \pi \\
\therefore 2 M_{\mathrm{AB}} & =549,109 \pi \\
\frac{M_{\mathrm{AB}}}{\pi} & =274,554.5 \mathrm{~cm}
\end{aligned}
$$

Formula (26) for the case of $c=0$, ex-

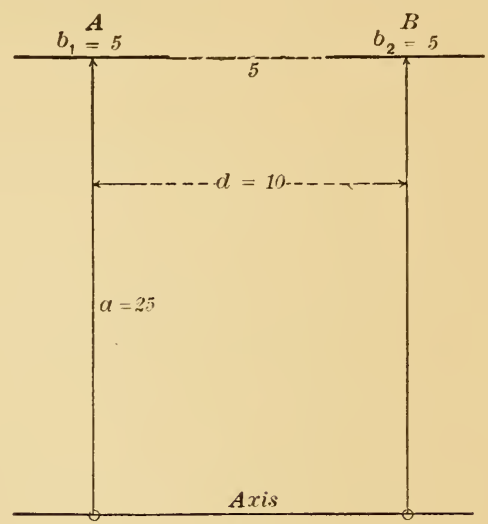

Fig. 17 tended to include two terms of the sixth and eighth degree (the fourth and fifth below) and a term depending on the differential coefficients of the eighth order (the tenth) is as follows: ${ }^{20}$

$$
\begin{aligned}
\frac{\Delta M}{\pi n^{2}} & =4 a\left[\frac{b^{2}}{\mathrm{I} 2 d^{2}}+\frac{b^{2}}{32 a^{2}}\left(\log \frac{8 a}{d}-\frac{\mathrm{II}}{6}\right)-\frac{\mathrm{I} 5 b^{2} d^{2}}{\mathrm{IO} 24 a^{4}}\left(\log \frac{8 a}{d}-\frac{97}{60}\right)\right. \\
& +\frac{\mathrm{I} 75 b^{2} d^{4}}{2(\mathrm{I} 28)^{2} a^{6}}\left(\log \frac{8 a}{d}-\frac{54}{35}\right)-\frac{3675 b^{2} d^{6}}{(\mathrm{I} 28)^{3} a^{8}}\left(\log \frac{8 a}{d}-\frac{3793}{2520}\right) \\
& \left.+\frac{b^{4}}{960 a^{2} d^{2}}+\frac{b^{4}}{60 d^{4}}-\frac{b^{4}}{\mathrm{IO} 24 a^{4}}\left(\log \frac{8 a}{d}-\frac{\mathrm{I} 87}{60}\right)+\frac{b^{6}}{\mathrm{I} 68 d^{6}}+\frac{b^{8}}{360 d^{8}}\right]
\end{aligned}
$$

The following is the calculation of $\Delta M$ for the two single layer coils above, considered as current sheets :

$$
a=25, \quad b=5, \quad d=\text { I0 } \quad \log _{e} \frac{8 a}{d}=2.99573
$$

${ }^{19}$ Bulletin of Bureau of Standards, 2, p. 136; 1906.

${ }^{20}$ Rosa, this Bulletin, p. $35 \mathrm{I}$, equation (5I). 


$$
\begin{aligned}
& \text { (I) } \frac{b^{2}}{\mathrm{I} 2 d^{2}} \\
& =\frac{25}{\mathrm{I} 200} \\
& =.0208333 \\
& \text { (2) } \frac{b^{2}}{32 a^{2}}\left(\log \frac{8 a}{d}-\frac{\mathrm{II}}{6}\right) \\
& =\frac{25 \times 1.1624}{20000} \\
& =.0014530 \\
& \text { (4) } \frac{\mathrm{I} 75 b^{2} d^{4}}{2(\mathrm{I} 28)^{2} a^{6}}\left(\log \frac{8 a}{d}-\frac{54}{35}\right)=\frac{\mathrm{I} 75 \times 250000 \times 1.45}{2(\mathrm{I} 28)^{2} 25^{6}}=.0000080 \\
& \text { (6) } \frac{b^{4}}{960 a^{2} d^{2}} \\
& =\frac{2^{2}}{960 \times \frac{5^{2}}{25^{2}} \times 100}=.0000104 \\
& (7) \frac{b^{4}}{60 d^{4}} \\
& =\frac{625}{60 \times 10,000} \\
& =.00104 \mathrm{I} 7 \\
& \begin{array}{l}
\text { (8) } \frac{-b^{4}}{\text { IO } 24 a} \\
\text { (9) } \frac{b^{6}}{\operatorname{I} 68 d^{6}}
\end{array} \\
& =+\frac{625 \times 0.121}{1024 \times \overline{625}^{2}} \\
& =.0000002 \\
& =\frac{1}{168.2^{6}} \\
& =.0000930 \\
& \text { (I0) } \frac{b^{8}}{360 d^{8}} \\
& =\frac{1}{-360 \times 2^{8}} \\
& \begin{array}{r}
=.0000108 \\
+.0234504
\end{array} \\
& \text { (3) }-\frac{\mathrm{I} 5 b^{2} d^{2}}{\mathrm{IO} 24 a^{4}}\left(\log \frac{8 a}{d}-\frac{97}{60}\right)=\frac{37,500 \times \mathrm{I} \cdot 379}{\mathrm{I0} 24 \times 25^{4}} \\
& =-.0001293 \\
& (5)-\frac{3675 b^{2} d^{6}}{(128)^{3} a^{8}}\left(\log \frac{8 a}{d}-\frac{3793}{2520}\right)=\ldots \ldots=\frac{-.0000004}{-.0001297} \\
& \text { Sum of ro terms }=+.0233207 \\
& 4 a n^{2}=250,000 \\
& \therefore \frac{\Delta M}{\pi} \ldots=5,830.2 \mathrm{~cm}
\end{aligned}
$$

By formula (I) we find $M_{0}$, the mutual inductance of two circles at the centers of these current sheets, for which $a=25, d=\mathrm{IO}$, to be $268,721.3 \mathrm{~cm}$. We thus have

$$
M=M_{0}+\Delta M=\pi(268,72 \mathrm{I} \cdot 3+5,83 \mathrm{O} .2) \text { or } \frac{M}{\pi}=274,55 \mathrm{I} \cdot 5 \mathrm{~cm}
$$

This is less than the value found above from the self inductance formula by $3 \mathrm{~cm}$, which is about one part in roo,000. This very 
close agreement in the results of two entirely independent formulæ is very satisfactory.

We have also calculated $\Delta M$ for this case by formula (30) putting $c=0$. The result is as follows:

$$
\frac{\Delta M}{\pi}=4 a n^{2} \times .0232 \mathrm{I} 7=5,804.2
$$

This is a little less than the value found by (34), as we should expect, as (30) does not take account of the sixth and eighth differentials, as does (34). We can, however, pick out these two terms from (34) and add them to (30) and see what difference remains in the results. From the above calculation we see that these two terms (the ninth and tenth of 34 ) amount to .0001038 $\times 4 a n^{2}$. Adding to the result by $(30)$ we have

$$
\frac{\Delta M}{\pi}=250,000 \times .0233208=5,830.2 \mathrm{~cm}
$$

which is exactly the value found by (34). The remaining discrepancy of one part in a hundred thousand represents the terms depending on differential coefficients of order higher than the eighth. If the coils were farther apart or narrower, these higher terms would be still smaller. When they are nearer or broader they are more important. For two current sheets $10 \mathrm{~cm}$ wide and $5 \mathrm{~cm}$ between there is a discrepancy of one part in 20,000 due to these terms of degree higher than the eighth. Such cases (where the coils are close together) should of course be computed by (33) rather than (34) for the highest accuracy, but (34) is more convenient and amply accurate for most purposes.

\section{EFFECT OF INSULATION ON THE WIRE.}

We have so far assumed in calculating $\Delta M$ that the current is uniformly distributed over the cross section of the coil. How much is $\Delta M$ altered if the current flows through round wires covered by insulation? We have found above that $\Delta M$ for a pair of coils, A, $\mathrm{B}$, of section $4 \times \mathrm{I} \mathrm{cm}$ and $\mathrm{ro} \mathrm{cm}$ between centers amounts to I. 38 where $M$ is 108.87 . That is, the mutual inductance is $1.3 \%$ greater when the current is distributed uniformly over the section than 
when it is concentrated at the centers of section $\mathrm{O}_{1} \mathrm{O}_{2}$. If, however, the current flows in four circles at the centers of the four small squares making up the section, as in $\mathrm{A}_{2} \mathrm{~B}_{2}$, the excess for uniform distribution is only .or 3 , or about a hundredth of I per cent. If the current were to flow through round wires of considerable section, as indicated in $\mathrm{A}_{2} \mathrm{~B}_{2}$, the difference would be still smaller. Hence four heavily insulated wires, with an exterior diameter of $\mathrm{I} \mathrm{cm}$, make up a coil which is equivalent to a winding of square wires with infinitesimal insulation to within about one part in ten thousand in the value of $M$, for the dimensions of this example.

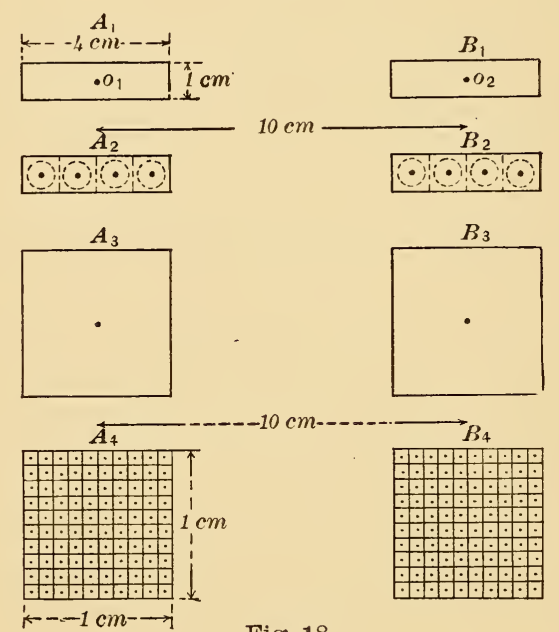

Fig. 18

If the cross section is square, $\mathrm{I} \mathrm{cm}$ on a side, and $a$ is $25 \mathrm{~cm}$ and $d$ is Io $\mathrm{cm}, \Delta M$ is, as in the last case, .0I3, when $M_{0}$ is 108.87 , or about one part in Io,ooo; that is, the difference in $M$ when the current is concentrated at the center in one case and uniformly distributed over the square in the other is a little more than one in Io,00o. If, however, the section be filled with insulated wire of one millimeter diameter, so that there are IOO small squares, $\Delta M$ for the whole coil will be proportional to the mean value of $\Delta M$ for the small squares. This will be less than I per cent of the former value, as the correction is proportional to $b^{2}$ and $b^{4}$. Hence the difference in $M$ between the case of uniform distribution of current 
over the section and having it concentrated in Ioo insulated wires of $100 \mathrm{~mm}$ diameter is certainly less than one part in a 111ilion of $M$, for the given case. We may be sure therefore that in every practical case we are justified in employing our formulæ for $\Delta M$, even though the current is not actually distributed over the entire cross section of the wire.

In taking the dimensions of the section we must take the entire space filled by the wire, including the insulation, on the wires forming the outer layers. That is, if the width of the section is made so that a given number of wires fills it closely, we should take $b$ as the total width of the channel; $c$ will be half the difference between the diameter before and after winding.

In order to reduce the uncertainty of the position of the wire and increase the uniformity of the winding, enameled-covered wire, which has a very thin and uniform covering, should be used. The effect of a slight lack of uniformity in winding parallel to the axis is eliminated by interchanging the coils in measuring the mutual inductance. The effect of a lack of uniformity radially is eliminated by determining the equivalent radius experimentally by comparison with a single layer coil.

The effect of slight errors in the values of $b$ and $c$ will be least when the section is square. The section should of course be as small as is consistent with obtaining a suitable value of $M$ and keeping the resistance within reasonable limits.

The close agreement of independent formulæ when applied to particular cases inspires confidence in the formulæ, and it is for this reason that we have carried ont the calculations in the above examples further than would be strictly necessary for use in experimental work. We think that, by properly choosing the dimensions of the coils, and decomposing them in the calculations into two or more parts when the sections are relatively large, that there is no difficulty in calculating the mutual inductances of a pair of coils of equal radii to one part in 50,000 or 100,000. By the methods of Bosscha, ${ }^{21}$ Rayleigh, ${ }^{22}$ and Lyle ${ }^{23}$ the mean radius can be accurately

${ }^{21}$ Bosscha: Pogg. Annalen, 93, p. 402 ; 1854 .

${ }^{22}$ Rayleigh, Sci. Papers, Vol. II, p. I 84.

${ }^{23}$ Lyle, Phil. Mag., 3, p. 310; 1902.

I6360-07-7 
determined with reference to a standard which is capable of very precise direct measurement. The distance apart can also be determined with very great accuracy, so that the possibilities of accuracy in the measurements as well as in the experimental determinations of the mutual inductance justify the use of very accurate formulæ in the calculations. Thus in absolute determinations of resistance by the measurement of self-inductance, results may be obtained not only by single layer coils but also by means of coaxial coils of several layers. 